\title{
A REVISION OF THE FORMS OF THE GREAT BLUE HERON (ARDEA HERODIAS LINNAEUS).
}

\author{
By Harry C. Oberholser, \\ Of the Biological Survey, United States Department of Agriculture.
}

Large birds, as a rule, do not seem to prove generally attractive to the modern systematic reviser, chiefly because of the usual difficulty of gathering together a satisfactory series, and of handling the material in making comparisons. The great blue heron, Ardea herodias Linnæus, like many other large species of North American birds, has been in much need of careful systematic study, and the results in the following pages are offered as an attempt at such revision. Previous literature bearing on the subject is confined to descriptions of new forms, and to other short papers. Like the writer's recent review of Butorides virescens and its races, ${ }^{1}$ the present paper was undertaken at the suggestion of Mr. H. W. Henshaw, chief of the Biological Survey, as an aid in working out the ranges of the forms of this species for a forthcoming bulletin by the Biological Survey on the distribution and migration of the North American herons.

Notwithstanding the difficulty of obtaining material, it has been possible to examine altogether 221 specimens of Ardea herodias, including the types of all its valid races except two, Ardea herodias herodias and Ardea herodias lessonii. This material comprises the entire collections of the United States National Museum, with that of the Biological Survey; the American Museum of Natural History; the Academy of Natural Sciences of Philadelphia, with those of Mr. Witmer Stone and Mr. H. W. Fowler; the collection of Dr. Jonathan Dwight, jr.; also a number of specimens from the Museum of Comparative Zoology at Cambridge, Massachusetts; from Messrs. A. E. and O. Bangs; the Field Museum, of Chicago; Mr. John E. Thayer; and Mr. Edward A. Preble. In addition, Mr. Joseph Grinnell very kindly made some comparisons of specimens in the Museum of Vertebrate Zoology of the University of California, also sent measurements of a number of specimens; and Mr. G. Willett has furnished information concerning the occurrence of the species on the Santa

1 Proc. U. S. Nat. Mus., vol. 42, 1912, pp. 529-576. 
Barbara Islands, California. To all of these institutions and individuals the writer wishes to express his obligation; also particularly to Dr. Charles W. Richmond, the Assistant Curator of Birds in the United States National Museum.

The geographical range of Ardea herodias, as a species, extends from southern Alaska and southern Canada, south through the United States, Mexico, Central America, and the West Indies, to northern South America. Although the available specimens from some regions are few, there are apparently 10 recognizable races, which subsequent investigation of abundant material may increase by one or two. There are already six current forms, so that the present investigation has resulted in the addition of four. The great blue heron lives in almost any sort of country, forest or open, desert or humid, if it has only the one requisite-water, from which it obtains the major portion of its food. It breeds usually in colonies, in trees or on the ground, and doubtless wanders far in search of food, along the shores, shallows, and muddy banks of streams, lagoons, and ponds, which are its favorite hunting grounds. Too little is known of the details of distribution to permit a very decided opinion on the life zone affinities of the various subspecies, but from what we know it does not appear that, except in a general way, they conform very well to accepted zonal boundaries, as suitable nesting sites are often the controlling influence. Consequently, the attempt to give them some such status must be taken with proper reservation. Most of the races are more or less migratory, though some of this movement is doubtless the well-known preæstival and postæstival wandering in which herons so commonly indulge. Two forms, Ardea herodias cognata, and Ardea herodias otigista, ${ }^{1}$ with probably also Ardea herodias sanctilucae, and possibly Ardea herodias fannini, are sedentary, or at least do not pass beyond their ascribed breeding areas. As in so many other wide-ranging species, some of the far-separated subspecies resemble each other much more than they do the adjacent forms with which they must bear much closer phylogentic relationship. For instance, the West Indian race, Ardea herodias adoxa, ${ }^{2}$ is much more nearly like Ardea herodias herodias than like the intervening Ardea herodias wardi; Ardea herodias cognata, from the Galapagos Islands, very much more closely resembles Ardea herodias treganzai, from the southwestern United States, than it does Ardea herodias lessonii ${ }^{3}$ from Mexico; Ardea herodias otigista ${ }^{1}$ and Ardea herodias hyperonca ${ }^{4}$ are both much more like Ardea herodias herodias from the eastern United States than like the interposed Ardea herodias treganzai; while the Mexican Ardea herodias lessonii ${ }^{3}$ is closest in appearance to Ardea herodias fannini of British Columbia, instead of to the 
adjoining Ardea herodias treganzai, of Arizona, or the also intervening Ardea herodias hyperonca ${ }^{1}$ of California. All but three of the races, Ardea herodias sanctilucae, Ardea herodias cognata, and Ardea herodias oligista, ${ }^{2}$ have a range of considerable, in some cases, of wide extent, as seems to be often the case with large birds which wander about over a large area. The size of the various races does not seem to correspond with any regularity to geographic conditions, for while the southernmost form, Ardea herodias cognata, of the Galapagos Islands, is of small size, the smallest race is Ardea herodias oligista, ${ }^{2}$ of the Santa Barbara Islands; while on the adjacent California mainland is one of the largest, Ardea herodias hyperonca, ${ }^{1}$ beyond which, in the northernmost subspecies, Ardea herodias fannini, lives a bird of only medium size. Furthermore, the Floridian Ardea herodias wardi is larger than either Ardea herodias adoxa ${ }^{3}$ from the West Indies, or Ardea herodias herodias from the northeastern United States.

The adult female of Ardea herodias is identical in color with the male, but averages smaller. Individual variation is slight, and consists principally in size, and in the color of neck and back. Seasonal change in plumage is likewise insignificant, probably because of the little wear to which the plumage is subject; and, though the neck sometimes becomes a little lighter in late summer, the back and other parts seemingly undergo little or no change. Apparently soon after the young are hatched, however, the color of the bare skin around the eye in the adult changes to yellowish green, and the maxilla becomes almost entirely dusky olive, leaving only its tomia and the mandible yellowish.

The young bird in first complete (i. e., juvenal) plumage differs considerably from the adult in having the whole pileum and upper sides of head dull, dark brown or brownish black; the entire neck and lower half of the sides of head much spotted or washed with buff, ochraceous, tawny, or chestnut; ground color of neck dull gray without any drab tinge; upper parts of body, wings, and tail, duller, more brownish, the long, plumelike development of dorsal and scapular feathers lacking; superior wing-coverts tipped or terminally spotted with ochraceous or tawny; edge of wing paler and more mixed with white; thighs somewhat lighter; breast and abdomen white, streaked broadly with gray and slate color, and more or less mixed with cinnamon rufous or buff; jugular plumes wanting; and the tufts on sides of breast gray striped with white; iris gamboge yellow; eyelids and bare horizontal lore space light apple green; maxilla black or blackish, the tomium brownish or horn color; mandible pale pea green, passing into clear horn yellow on the ter- 
minal half; tibia and soles of toes apple green; remainder of legs and feet black. From this stage the bird gradually passes into the adult plumage, and specimens with all sorts of combinations of adult and young plumages are to be found. Some individuals in juvenal plumage are smaller than adults, but the young bird becomes fully grown before assuming the adult livery.

The diagnostic characters most useful in separating the various races of Ardea herodias are size, together with the shade of color on the neck and upper parts. The posterior lower surface is practically the same in all the forms. The juvenal plumage in most cases reflects the color differences of the adult, though usually to a less degree. All measurements are given in millimeters, and have been taken as explained in the writer's paper on Butorides virescens. ${ }^{1}$ In the averages none but typical specimens have been used, whenever such could be obtained. Furthermore, in the tables of detailed measurements every specimen used in the diagnostic averages is indicated; and all other than adults are properly noted. The names of colors employed are based on Mr. Robert Ridgway's Nomenclature of Colors. ${ }^{2}$

In working out the races of Ardea herodias it became necessary to determine as far às possible the status of Ardea occidentalis Audubon, ${ }^{3}$ Ardea wardi Ridgway, ${ }^{4}$ and the much discussed Ardea wrirdemannii Baird, ${ }^{5}$ all from Florida, since this problem's solution bears directly on the proper name for the race of Ardea herodias inhabiting Florida. The detailed evidence concerning these birds will be presented in another paper.

\section{ARDEA HERODIAS HERODIAS Linnæus.}

[Ardea] herodias Lrnnæus, Syst. Nat., ed. 10, vol. 1, 1758, p. 143 (America) (based on "Ardea fusca canadensis," Edwards [="The Ash-colour'd Heron from North America,'”Edwards], Nat. Hist. Birds, vol. 3, 1750, p. 135, pl. 135; vol. 4, 1751, p. 245 [Hudson Bay]; and, with a query, on "Ardea cristata maxima americana," Catesby, Nat. Hist. Carolina, Fla., and Bahama Is., vol. 2, Appendix, 1753, p. 10, pl. 10 [Virginia]).

[Ardea] hudsonias Linn.eus, Syst. Nat., ed. 12, vol. 1, 1766, p. 238 (Hudson Bay) (based on "Ardea americae septentrionalis"' [ ="The Ash-colour'd Heron from North America'], Edwards, Nat. Hist. Birds, vol. 3, 1750, p. 135, pl. 135 [Hudson Bay]; and on "Ardea freti hudsonis," Brisson, Ornith., vol. 5, 1760, p. 407 [Hudson Bay]).

Chars. subsp.-Size rather small (compared with other races); neck and upper parts moderately dark in color.

Description.-Adult male, No. 153329, U.S.N.M.; Brewerton, New York, April 30, 1881; Edwin M. Hasbrouck. Sides of crown, and

${ }^{1}$ Oberholser, Proc. U. S. Nat. Mus., vol. 42, 1912, p. 533.

2 Ridgway, Nomenclature of Colors for Naturalists, Boston, 1886.

${ }^{3}$ A rdea occidentalis Audubon, Birds Amer. (folio), vol. 3, No. 57, 1835, pl. 281 (Florida Keys).

4 Ardea wardi Ridgway, Bull. Nutt. Orn. Club, vol. 7, January, 1882, p. 5 (Oyster Bay, Florida).

- Ardea würdemannii Baird, Rep. Expl. and Surv. R. R. Pac., vol. 9, 1858, p. 669 (southern Florida). 
long, slender, pointed, occipital crest black; forehead, center of crown, short vertical crest, superciliary stripe, malar region, chin, and middle of upper throat, white; sides of head and throat drab gray; neck all around deep drab gray, the middle line of foreneck streaked with black, white, and cinnamon rufous; upper surface, including tail, tertials, innermost secondaries, and superior secondary coverts, slate gray, the long, narrow, plumaceous feathers of back and scapulars paler and glaucous, the median coverts and outer webs of greater coverts paler gray, the rectrices somewhat brownish and distally darker, inclining to slate color or blackish slate; primaries, secondaries (except the innermost), primary coverts, and alula, blackish slate, slate black or dull black; jugulum smoke gray, medially white, streaked broadly with black, dull brownish slate, narrowly with cinnamon rufous and pale cinnamon, the long, narrow, pointed plumelike feathers terminally pale drab gray, smoke gray or whitish; a tuft of black, partly white-striped feathers on each side of the breast; back of this a small patch of cinnamon rufous; sides and flanks slate gray; breast and abdomen black, broadly streaked with white; lower tail-coverts white; thighs and edge of wing cinnamon rufous, the latter shading to chestnut along the secondaries; lining of wing slate color, the axillars slate gray.

In full spring plumage the iris is chrome yellow; the bare orbital space cobalt blue; bill wax yellow, brighter on the mandible, the culmen sometimes brownish; legs black or dark brownish, the tibia usually more brownish; soles of toes dull yellowish.

Measurements.-Length (in flesh), 1067-1270 mm.; extent of wing, 1790-1920 mm.; weight, $5-8$ pounds.

Male: ${ }^{1}$ Wing, 441-280 (average, 462.7) mm.; tail, 167-187 (176.6); exposed culmen, 123-151.5 (139.5); height of bill at base, 23.5-31.3 (27.6); tarsus, 167-205 (183.6); middle toe, 93-114.5 (106.5).

Female: ${ }^{2}$ Wing, 433-471 (451.2) mm.; tail, 159-184 (173.7); exposed culmen, 127-146 (137); height of bill at base, 24.5-28.5 (26.7); tarsus, 157-194 (175.4); middle toe, 93-115 (102.4).

Type-locality.-Hudson Bay, Canada.

Geographical distribution.--Eastern United States and southern Canada: In summer chiefly the Upper Austral, Transition, and Canadian zones, north to Anticosti Island and Godbout, northeastern Quebec; Lake Temiskaming, central Quebec; Moose Factory and Rat Portage, northern Ontario; Shoal Lake and Duck Mountain, central Manitoba; Osler, southern Saskatchewan; and Edmonton, central Alberta; west to southeastern British Columbia (probably); North Dakota; South Dakota; and probably eastern Nebraska;

1 Ten specimens, from New York, Pennsylvania, New Jersey, Virginia, North Carolina, and Florida. 2 Twelve specimens, from New York, New Jersey, Connecticut, Maryland, South Carolina, Illinois, and Tamaulipas. 
south to southern Alberta (probably); southern Saskatchewan (probably); eastern Nebraska (probably); central Iowa; central Illinois; central Indiana; eastern Tennessee; and near Charleston, central eastern South Carolina; east to the Atlantic coast of the United States and of southern Canada, and to the Bermuda Islands. ${ }^{1}$ Winters from Florida, northern Tamaulipas, and Texas, north to New York State; also on the Bermuda Islands. In migration or in winter it wanders west to Prospect Lake, British Columbia.

The present subspecies appears to be very uniform over its entire range, at least the specimens examined so indicate. There seems to be little if any difference, in either size or color, between examples from New York and Connecticut and those from North and South Carolina. A specimen from Castleberry, Alabama, taken November 10, 1911, is large, but very dark, even for Ardea herodias herodias, and is nearer this than to Ardea herodias wardi. Another bird, taken at the same place on November 18, 1911, is typical Ardea h. herodias. Two birds from Mount Carmel, Illinois (U.S.N.M. Nos. 84577 and 84578), are slightly larger and slightly lighter above than typical examples of Ardea herodias herodias, inclining thus toward Ardea herodias wardi, but they are much nearer the present form. As these two were not breeding birds they were doubtless sojourners from another region, since the breeding form of this locality is Ardea herodias wardi. The breeding bird of Saskatchewan and Alberta is probably Ardea herodias herodias, although no specimens are at hand. An immature individual from Prospect Lake, British Columbia, September 18, 1896 (No. 73573, Amer. Mus. Nat. Hist.), is just like immature Ardea herodias herodias, so far as I can see, though it may possibly be an intermediate between Ardea herodias treganzai and Ardea herodias fannini. What is the breeding form of the Bermuda Islands is a little uncertain, as I have examined no specimens from there, but the probabilities favor its proper reference to Ardea herodias herodias.

All specimens from Florida are, of course, only winter residents, and this State seems to be a regular winter home for members of this subspecies. It winters, however, with apparent regularity also north to New York State, as there are several winter records of specimens from the States of New York, New Jersey, and Virginia. A single bird (No. 183328, U.S.N.M.) taken January 27, 1902, at Camargo, Tamaulipas, Mexico, on the Rio Grande, represents the southwestern limit of its known range. There is no certain record of its occurrence in Louisiana, though it doubtless occurs there during winter. A bird taken September 17, 1886, by naturalists of the U. S. Fish Commission steamer Albatross, at sea about 130 miles off the coast of New Jersey (lat. $38^{\circ} 29^{\prime} \mathrm{N}$.; long. $71^{\circ} 58^{\prime}$ W.), shows how the species sometimes wanders. 
The original description of Ardea herodias ${ }^{1}$ applies without doubt to the form of great blue heron inhabiting the northeastern part of North America. Linnæus' description was based on the "Ardea fusca canadensis," or "The Ash colour'd Heron from North America" of Edwards; ${ }^{2}$ and with a query on the "Ardea cristata maxima americana" of Catesby; ${ }^{3}$ both of which may be unquestionably referred to the great blue heron. Since Linnæus gives only "America" as the locality of his species, and since he apparently considered the identity of Catesby's bird doubtful, the type locality should, therefore, be Hudson Bay, from Edwards. The Ardea hudsonias of Linnæus ${ }^{4}$ is a strict synonym of Ardea herodias, since it has in part the same basis-"Ardea americae septentrionalis" = "The Ash colour'd Heron from North America" of Edwards, ${ }^{5}$-and also the "Ardea freti hudsonis" of Brisson, ${ }^{6}$ both of which refer to the bird from Hudson Bay.

In all, 76 specimens of this form have been examined, from the following localities, breeding records being indicated by an asterisk:

British Columbia.-Prospect Lake.

Nova Scotia.-Newport.*

Quebec.-Tadousac.

Alabama.-Castleberry.

Connecticut.-Liberty Hill; Rockville.

District of Columbia.-Washington.*

Florida.-Gainesville; Oak Lodge (eastern peninsula opposite Micco) (Feb. 1); Lake Harney (Dec. 3).

Illinois.-Mount Carmel.

Iowa.-Winnebago County.*

Maine.-Island Falls; Brewer.

Maryland.-Sandy Spring;* Glen Echo.*

Massachusetts.-Dedham.

New Hampshire.-Hollis.

New Jersey.-Haddonfield; Timber Creek;* Salem County;* Five Mile Beach (Cape May County);* Cape May County; South Atlantic City; Atlantic City; Masonville; Mouth of Delaware River (Jan. 26); Atlantic Ocean off coast of New Jersey (lat. $38^{\circ} 29^{\prime}$ N.; long. $71^{\circ} 58^{\prime}$ W.).

New York.-Brewerton;* Springs (Dec. 20); Long Island (Dec. 28); Shelter Island; Ardsley (Westchester County); Lawrence County; Oakdale (Suffolk County); Consook Marsh, Highland Falls (Dec. 12); Rockaway (Dec. 14).

1 Linnæus, Syst. Nat., ed. 10, vol. 1, 1758, p. 143.

2 Nat. Hist. Birds, vol. 3, 1750, p. 135, pl. 135; vol. 4, 1751, p. 245

3 Nat. Hist. Carolina, Fla., and Bahama Is., vol. 2, Appendix, 1753, p. 10, pl. 10.

4 Syst. Nat., ed. 12, vol. 1, 1766, p. 238.

6 Nat. Hist. Birds, vol. 3, 1750, p. 135, pl. 135.

6 Ornith., vol. 5,1760 , p. 407. 
North Carolina.-Currituck (Jan. 25); Hatteras.

Pennsylvania.-Conneau tee Lake (Erie County); Holmesburg (Philadelphia);* Bristol (Bucks County).*

South Carolina.-Porcher's Bluff, Christchurch Parish.*

Virginia.-Cape Charles (Jan. 1); Strasburg; Dismal Swamp.*

Wisconsin.-Waupaca.

Tamaulipas.-Camargo.

Measurements of specimens of Ardea herodias herodias.

\begin{tabular}{|c|c|c|c|c|c|c|c|c|c|c|}
\hline Museum and No. & Sex. & Locality. & Date. & Collector. & $\stackrel{9}{\nexists}$ & స్త్̈ & 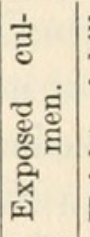 & 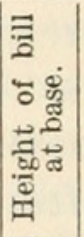 & 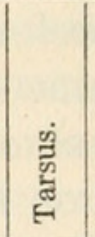 & 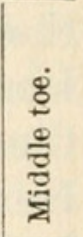 \\
\hline U.S.N.M. $153329^{1}$.. & Male...... & Brewerton, & Apr. 30,1881 & E. M. Has- & $\begin{array}{r}m m \\
465\end{array}$ & $\begin{array}{l}m m \\
180\end{array}$ & $\begin{array}{l}m m \\
144\end{array}$ & $\begin{array}{l}m m \\
30\end{array}$ & $\begin{array}{c}m m \\
175\end{array}$ & $\begin{array}{c}m m \\
109\end{array}$ \\
\hline H. W. Fowler $1316^{1}$ & Male juv. ${ }^{2}$ & Holmesburg & July 28,1897 & J. Walls..... & 480 & 182 & 141 & 23.5 & 205 & 114.5 \\
\hline H. W. Fowler $1071^{1}$ & Male. & $\begin{array}{l}\text { B ris t o l, } \\
\text { Bucks Co., }\end{array}$ & Apr. 9,1896 & W. Hall..... & 447 & 176 & 123 & 24.5 & 172 & 99 \\
\hline U.S.N.M. $156226^{1} .$. & Male juv.2 & Pa. & Sept. 3,1895 & P. W. Roth.. & 465 & 175 & 134 & 27 & 188 & 105 \\
\hline A.N.S.Phila. 337281 & $\ldots d o^{2}$. & Haddonfield, & Oct. 29,1879 & S.N.Rhoads. & 448 & 167 & 133 & 27 & 176 & 93 \\
\hline H. W. Fowler $1315^{1}$ & $\ldots \mathrm{do}^{2}$ & $\begin{array}{l}\text { Tim ber } \\
\text { Creek. N.J. }\end{array}$ & July 16,1897 & F. Schluter.. & 464 & 172 & 136 & 31.3 & 188.5 & 111 \\
\hline Am. Mus. N. H. & Male & $\begin{array}{l}\text { Currituck, } \\
\text { N.C. }\end{array}$ & Nov. 27,1897 & J.C. Barron . & 468 & 180.5 & 151.5 & 27 & 197 & 109 \\
\hline Am. Mus. N. H. & ... do.. & Hatteras,N.C. & Mar. 6,1900 & J.H. Batty.. & 441 & 173 & 138 & 27 & 167 & 100 \\
\hline E. A. Preble $812^{1} \ldots$ & ... do:. & $\begin{array}{l}\text { Strasburg, } \\
\text { Va. }\end{array}$ & Apr. 28,1900 & E. A. Preble. & 473 & 187 & 149 & 31 & 182 & 112 \\
\hline A.E. and O.Bangs & ... do. & $\begin{array}{l}\text { Oak Lodge } \\
\text { (e as tern } \\
\text { peninsula } \\
\text { opposite } \\
\text { Micco), Fla. }\end{array}$ & Feb. 1,1895 & O. Bangs.... & 476 & 173 & 145.5 & 27.5 & 185 & 112.5 \\
\hline J. D wight $15275^{1} \ldots$ & $\underset{\text { juv. }^{2}}{\text { Female }}$ & $\begin{array}{l}\text { Springs, } \\
\text { N.Y. }\end{array}$ & & & 438 & 159 & & 25 & 168 & 101 \\
\hline $\begin{array}{l}\text { J. Dwight } 15243^{1} \\
\text { J. Dwight } 11571^{1}\end{array}$ & $\begin{array}{l}\ldots \text { do }^{2} \text {. } \\
\ldots \text { do }^{2} \text {. }\end{array}$ & Sheider...... Is- & $\begin{array}{l}\text { Dec. } 20,1905 \\
\text { Oct. } 1,1904\end{array}$ & W. W. Wor- & $\begin{array}{l}450 \\
433\end{array}$ & $\begin{array}{l}170 \\
166\end{array}$ & $\begin{array}{l}135.5 \\
131\end{array}$ & $\begin{array}{l}26.5 \\
24.5\end{array}$ & $\begin{array}{l}177 \\
174\end{array}$ & $\begin{array}{r}100 \\
95\end{array}$ \\
\hline J. D wight $6777^{1}$.. & Female. & $\begin{array}{l}\text { land, N.Y. } \\
\ldots \text { do } \ldots . . .\end{array}$ & & $\begin{array}{l}\text { thington. } \\
\text {..... do........ }\end{array}$ & & 166 & & 27 & 160 & 100 \\
\hline$\underset{70305.3}{\operatorname{Am} .}$ Mus. N. H. & $\begin{array}{l}\text { Female } \\
\text { juv. }\end{array}$ & $\begin{array}{c}\text { A r d s le y } \\
\text { Westchester } \\
\text { ter Co.,N.Y. }\end{array}$ & Oct. 13,1898 & C. Travis.... & 425 & 163 & 121.5 & 26 & 162.5 & 100 \\
\hline Am. Mus. N. H. & $\ldots$ do & $\begin{array}{c}\text { Lawrence } \\
\text { Co., N.Y. }\end{array}$ & Aug.27, 1895 & $\begin{array}{l}\text { O. A. Schroe- } \\
\text { der. }\end{array}$ & 450 & 169 & 120 & 24.5 & 172.5 & 105 \\
\hline A.E. and O. Bangs & Female.. & $\begin{array}{l}\text { Liberty Hill, } \\
\text { Conn. }\end{array}$ & Apr. 10,1895 & & 463 & 178 & 133 & 28.5 & 167 & 99 \\
\hline H. W. Fowler $1317^{3}$ & $\begin{array}{l}\text { Female } \\
\text { juv. }\end{array}$ & $\begin{array}{l}\text { Holmesburg } \\
\text { (Phila.)Pa. }\end{array}$ & Aug.13, 1897 & J. Walls.... & 464 & 179 & 135 & 29.5 & 5195 & 106 \\
\hline W. Stone $1974^{1} \ldots$. & Female. & $\begin{array}{l}\text { Salem Coun- } \\
\text { ty, N. J. }\end{array}$ & May 7,1896 & $\begin{array}{l}\text { D. N. McCad- } \\
\text { den. }\end{array}$ & 459 & 182 & 138.5 & 27 & 181 & 103 \\
\hline A.N.S.Phila. $26026^{1}$ & ..do. & $\begin{array}{l}\text { Five Mile } \\
\text { Beach,Cape } \\
\text { M a y C o., } \\
\text { N. J. }\end{array}$ & Apr. 14,1879 & $\begin{array}{l}\text { W. L. Ab- } \\
\text { bott. }\end{array}$ & 471 & 184 & 136.5 & 26.3 & 188.5 & 103 \\
\hline U.S.N.M.79367 & & $\begin{array}{l}\text { s } \underset{\text { spring, }}{\text { a }} \text { d d. } \\
\text { d. }\end{array}$ & Mar. -, 1880 & R. Ridgway. & 435 & 164 & 140 & 27 & 157 & 93 \\
\hline M.C.Z. $42543^{1} \ldots$. & & $\begin{array}{l}\text { Currituck, } \\
\text { N.C. }\end{array}$ & Jan. 25,1887 & W.S. Bryant & 460 & 182 & 145 & 28.5 & 5194 & 115 \\
\hline U.S.N.M. $222008^{1}$. & ...do. & $\begin{array}{l}\text { P or c h e r's } \\
\text { Bluff,Christ- } \\
\text { church Par- } \\
\text { ish, S. C. } \\
\text { Mount Car- }\end{array}$ & Apr. 28, 1911 & E.A.Mearns. & 455 & 172 & 146 & 27.5 & 178 & 111 \\
\hline U.S.N.M. $183328^{1}$. & ... do. & $\begin{array}{l}\text { Ca mer, a r g o, } \\
\text { Tamaulipas } \\
\text { Mex. }\end{array}$ & Jan. 27, 1902 & $\begin{array}{l}\text { E. A. Gold- } \\
\text { man. }\end{array}$ & 465 & 180 & 136 & 26 & 180 & 101 \\
\hline
\end{tabular}

1 Used in measurement averages on p. 535.

2 Full grown, but in juvenal plumage.

8 Not full grown. 
Measurements of specimens of Ardea herodias herodias - Continued.

\begin{tabular}{|c|c|c|c|c|c|c|c|c|c|c|}
\hline Museum and No. & Sex. & Locality. & Date. & Collector: & $\stackrel{80}{\Xi}$ & 蔦 & 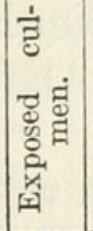 & 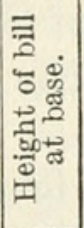 & 空 & 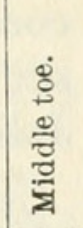 \\
\hline $\begin{array}{l}\text { Am. Mus. N. H. } \\
73573.1 \\
\text { Am. Mus. N. H. } \\
45753 \text {. }\end{array}$ & $\begin{array}{l}\text { Female } \\
\text { juv. }\end{array}$ & $\begin{array}{l}\text { Prospe e t } \\
\text { Lake, Brit. } \\
\text { Col. } \\
\text { Long Island, }\end{array}$ & Sept.18, 1896 & & $\begin{array}{r}m m \\
442\end{array}$ & $\underset{173}{m m}$ & $\begin{array}{l}\mathrm{mm} \\
123 \\
139\end{array}$ & $\begin{array}{l}m m \\
27.5 \\
25.5\end{array}$ & $\mid \begin{array}{l}m m \\
161 \\
159.5\end{array}$ & $\begin{array}{l}m m \\
89.5 \\
97\end{array}$ \\
\hline A.N.S.Phila.39659. & & $\begin{array}{l}\text { Mouth of Del- } \\
\text { aware Riv- } \\
\text { er [N. J.?] }\end{array}$ & Jan. 26, 1903 & W.D.Winsor & 432 & 175 & 140 & 25.5 & 173 & 98 \\
\hline $\begin{array}{l}\text { A.N.S.Phila. } 39660 . \\
\text { A.N.S.Phila.26025. }\end{array}$ & Jus & Cando..... & do $\ldots$..... & & $\begin{array}{l}436 \\
432\end{array}$ & 159 & $\left|\begin{array}{|c|}134.5 \\
132.5\end{array}\right|$ & 27 & 167 & 101.5 \\
\hline A.N.S.Phila.26025. & $\ldots$ de & Cape May & $\begin{array}{l}\text { Nov.27, } 1878 \\
\text { Sept }-1899\end{array}$ & $\begin{array}{l}\text { W. L. Ab- } \\
\text { bott. }\end{array}$ & 432 & 164 & $\left|\begin{array}{l}133.5 \\
145.5\end{array}\right|$ & $\begin{array}{l}26 \\
27.5\end{array}$ & $\begin{array}{l}176.5 \\
193\end{array}$ & 94.5 \\
\hline $\begin{array}{l}\text { A.N.S.Phila.58251. } \\
\text { A.N.S.Phila. } 34916 \text {. }\end{array}$ & - & $\begin{array}{l}\text { S o u th At- } \\
\text { lantic City, } \\
\text { N.J. } \\
\text { Ma sonville, }\end{array}$ & Sept.-, 1899 & $\begin{array}{l}\text { C. W. Buv- } \\
\text { inger and J. } \\
\text { A.G.Rehn. } \\
\text { L. Rodgers.. }\end{array}$ & 451 & 180 & 134 & 25.5 & 160 & 103 \\
\hline A.N.S.Phila.48276. & Jux & $\begin{array}{l}\text { N.J. } \\
\text { Salem } \\
\text { N. J. }\end{array}$ & Feb. 6,1905 & W. Hughes.. & 445 & 179 & 125 & 25 & 176 & 101 \\
\hline W. Stone $1207 . . .$. & & $\begin{array}{l}\text { Atlantic } \\
\text { City, N.J. }\end{array}$ & Oct. 2,1892 & $\begin{array}{l}\text { I. N. De Ha- } \\
\text { ven. }\end{array}$ & 452 & 174 & 146 & 27.5 & 181 & 106.5 \\
\hline Am. Mus. N. H. & & $\begin{array}{l}\text { Cape Charles, } \\
\text { Va. }\end{array}$ & Jan. 1, 1901 & G. A. Smith. & 445 & 181 & 138 & 28.5 & $\mid 179.5$ & 5109 \\
\hline U.S.N.M.159980_.. & Ju & $\begin{array}{l}\text { D i s m a l } \\
\text { Swamp, Va. }\end{array}$ & June 20,1897 & $\begin{array}{l}\text { D. W. Pren- } \\
\text { tiss, jr. }\end{array}$ & 456 & 177 & 139 & 27.5 & 195 & 117 \\
\hline T.S.N.M.. & & $\begin{array}{l}\text { Castleberry, } \\
\text { Ala. }\end{array}$ & Nov.18, 1911 & W.Matthews & 464 & 181 & 129 & 28 & 179 & 102 \\
\hline $\begin{array}{l}\text { U.S.N.M. } \\
\text { A.N.S.Phila.26027. }\end{array}$ & Juv & $\begin{array}{c}\text { W in nebago } \\
\mathrm{Co} \text {, Iowa. }\end{array}$ & $\begin{array}{l}\text { Nov.10, } 1911 \\
\text { Aug.19, } 1879\end{array}$ & $\begin{array}{l}\text { W. L... A } \mathrm{Ab}- \\
\text { bott. }\end{array}$ & $\begin{array}{l}490 \\
435\end{array}$ & $\begin{array}{l}184 \\
171\end{array}$ & $\begin{array}{l}155 \\
126\end{array}$ & $\begin{array}{l}29.8 \\
26.5\end{array}$ & $\begin{array}{l}195 \\
169\end{array}$ & $\begin{array}{r}114 \\
99\end{array}$ \\
\hline
\end{tabular}

\section{ARDEA HERODIAS WARDI Ridgway.}

Ardea wardi Ridgway, Bull. Nutt. Orn. Club, vol. 7, January, 1882, p. 5 (Oyster Bay, Florida).

Chars. subsp.- Similar to Ardea herodias herodias, but decidedly larger in all its measurements; upper surface and neck lighter in color.

Measurements. - Total length (in flesh), 1295-1335 mm.; extent of wing, 1970-2090.

Male: ${ }^{2}$ Wing, 486-518 (average, 497.7) mm.; tail, 181-209 (191.5); exposed culmen, 146-167 (156.9); height of bill at base, 28.5-32 (30.8) ; tarsus, 195-232 (210.9); middle toe, 115-129 (119.9).

Female: ${ }^{3}$ Wing, 471-489 (477.2); tail, 173-192 (181.5); exposed culmen, 140-147 (143.7); height of bill at base, 27-30.5 (28.1); tarsus, 189-214 (205.4); middle toe, 111-123 (116.2).

Type-locality.-Oyster Bay, Florida.

Geographical distribution.--Southeastern United States to central Mexico: In summer chiefly the Lower Austral Zone, north to Hiltonhead, southeastern South Carolina; southern Georgia; southern Alabama; Knox County, southwestern Indiana; Mount Carmel, southeastern Illinois; Henry County, southeastern Iowa; and Kansas; west to Kansas; central Oklahoma; Gainesville and probably Gurley, 
Texas; south to the Florida Keys and the Gulf coast of the southern United States, from Florida to Brownsville, Texas; east to the Atlantic coast of South Carolina, Georgia, and Florida. Winters in Florida, southern Alabama, and Texas; also in Mexico, south to Ocotlan, Jalisco.

This is a well-characterized form, and is easily distinguishable from Ardea herodias herodias. Even in the juvenal plumage it is usually paler on neck and upper surface than is Ardea h. herodias. A series of breeding birds from a cypress swamp near the mouth of the White River, in the southwestern corner of Knox County, Indiana, and one specimen from Mount Carmel, Illinois (No. 72837, U.S.N.M.), just across the Wabash River, are smaller than Ardea herodias wardi from Florida, and in this seem to be rather closer to Ardea herodias herodias, yet in color they average much nearer Ardea herodias wardi. A single example from Henry County, southeastern Iowa (No. 12358, J. Dwight), and another from an unknown but probably eastern locality in Kansas (No. 72836, U.S.N.M.) are pale like Ardea herodias wardi, and also large, too large, in fact, for Ardea herodias herodias. The present race thus doubtless occupies the southern Mississippi Valley, including eastern Kansas, eastern Oklahoma, and eastern Texas. A breeding bird from Hiltonhead, southeastern South Carolina (No. 39031, U.S.N.M.), is like Ardea herodias wardi in color, but is smaller, and in this somewhat intermediate between Ardea herodias wardi and Ardea herodias herodias. The same remarks will apply also to a specimen from Ossabaw Island, Georgia (No. 11902, J. Dwight), taken November 29, 1904. This Hiltonhead, South Carolina, record doubtless represents about the northernmost limit of the breeding range of this subspecies on the Atlantic coast, for the breeding form about Charleston is Ardea herodias herodias. No specimens from Mississippi or from the central or northern portions of Georgia or Alabama have been available, but Ardea herodias wardi without doubt occupies in summer the southern part of all these States. Birds from Corpus Christi, Texas, seem to be identical in both size and color with those from Florida. The following comparative averages of millimeter measurements of specimens from various parts of the range of Ardea herodias wardi show what differences exist: 


\begin{tabular}{|c|c|c|c|c|c|c|}
\hline Localities. & Wing. & Tail. & $\begin{array}{l}\text { Exposed } \\
\text { culmen. }\end{array}$ & $\begin{array}{c}\text { Height } \\
\text { of bill } \\
\text { at base. }\end{array}$ & Tarsus. & $\begin{array}{c}\text { Middle } \\
\text { toe. }\end{array}$ \\
\hline Eleven males, from Florida and Georgia... & $\underset{499.1}{m m}$ & $\underset{192.3}{m m}$ & $\begin{array}{l}m m \\
158\end{array}$ & $\begin{array}{l}m m \\
31.1\end{array}$ & $\underset{211}{m m}$ & $\begin{array}{l}m m \\
119.8\end{array}$ \\
\hline Two males, from Toxas........... & 496 & 185.5 & 156.5 & 30 & 214.5 & 123 \\
\hline One male, from Jalisco, Mexico........... & 486 & .195 & 146 & 29 & 202 & 115 \\
\hline Six males, from southwestern Indiana..... & 476 & 182.2 & 145.7 & 27.8 & 183.4 & 107.6 \\
\hline One male, from southeastern Iowa ........ & 486 & 190 & 153 & 29 & 194 & 119 \\
\hline One male, from Kansas............ & 490 & 184 & 158 & 29 & 202 & 107 \\
\hline Seven females, from Florida.. & 477.2 & 181.5 & 143.7 & 28.1 & 205.4 & 116.2 \\
\hline 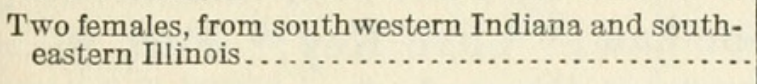 & 453 & 185.5 & 138 & 26.3 & 180.5 & 106 \\
\hline
\end{tabular}

This subspecies is evidently resident in Florida, as there is no West Indian or Bahaman specimen; and there are numerous winter records for Florida. It winters also north at least to southern Alabama, probably farther in the Mississippi Valley. An example which Messrs. E. W. Nelson and E. A. Goldman obtained at Ocotlan, Jalisco, Mexico, on December 25, 1902 (No. 184944, U.S.N.M.), seems to be undoubtedly of this form, and probably marks nearly or quite the southern limit of its winter range.

It breeds rather early in the year, at least in Florida; commonly in March (Suwanee River, Lafayette County, March 21, 1890, Mr. F. M. Chapman; Fort Gardner, March 9, 1901, Dr. E. A. Mearns); and, occasionally, at least, by the middle of January, for Mr. C. A. Smith found nest and eggs at Rutland, Florida, on January 15, 1890.

The form of Ardea herodias here called Ardea herodias wardi was first described by Mr. Ridgway as a full species, ${ }^{1}$ on the hypothesis that Ardea herodias, Ardea occidentalis, and Ardea wardi should be regarded as three distinct species, of which the last two were considered to possess both a white and a colored phase. That, however, Ardea wardi is but a subspecies of Ardea herodias, is evident from the regular intergradation with Ardea herodias herodias that takes place wherever the ranges of the two closely approach, from South Carolina to Illinois. Furthermore, pure white birds (Ardea occidentalis) occur only on the Florida Keys and the adjacent West Indian islands; and the so-called Ardea würdemannii only where the ranges of Ardea occidentalis and Ardea wardi overlap. The complicated and puzzling relationships of these four birds-Ardea occidentalis, Ardea würdemannii, Ardea wardi, and Ardea herodias - will be more fully discussed in a separate paper. Suffice it here further to state that the writer regards Ardea herodias as specifically distinct from Ardea occidentalis; Ardea wardi the Florida subspecies of Ardea herodias; ${ }^{2}$ Ardea

1 Ardea wardi Ridgway, Bull. Nutt. Orn. Club, vol. 7, January, 1882, p. 5.

2 See Chapman, Bull. Amer. Mus. Nat. Hist., vol. 14, A pril 15, 1901, pp. 88-89. 
occidentalis a distinct species; and Ardea würdemannii a hybrid between Ardea herodias wardi and Ardea occidentalis.

The writer has seen 60 specimens of Ardea herodias wardi from the subjoined localities, an asterisk marking records of breeding birds:

Alabama.-Orange Beach, Perdido Bay, Baldwin County (Jan. 28). Florida.-Drigg's Landing, Kissimmee River;* De Soto County (Dec. 8); Jacksonville; Lake Harney; eastern peninsula opposite Micco; near Micco;* San Mateo;* Palatka (Jan. 29); Gainesville; Sebastian (Jan. 29) ; Lake Hatch-ne-haw;* Clive Key;* Fort Bassinger; Punta Rassa (Mar. 10);* Suwanee River, Lafayette County;* Fort Gardner, Kissimmee River;* Clearwater; Seven Oaks; * Bremer Island, Lake Kissimmee;* Rutland; Tarpon Springs;* northern Brevard County; Amelia Island (Dec. 14); Amelia Island;* New Smyrna; Hernando County.*

Georgia.-St. Marys;* McIntosh County;* Ossabaw Island.

Illinois.-Mount Carmel.*

Indiana.-Cypress Swamp, near mouth of White River, southwestern Knox County.*

Iowa.-Henry County, 4 miles north of Hillsboro.

Kansas.-(No definite locality specified.)

South Carolina.-Hiltonhead.*

Texas.-Corpus Christi.*

Jalisco.-Ocotlan (Dec. 25).

Measurements of specimens of Ardea herodias wardi.

\begin{tabular}{|c|c|c|c|c|c|c|c|c|c|c|}
\hline Museum and No. & Sex. & Locality. & Date. & Collector. & $\stackrel{80}{\Xi}$ & 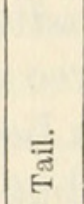 & 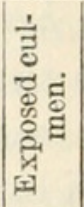 & 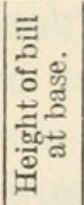 & 离 & 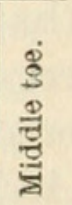 \\
\hline U.S.N.M. $77946^{1} \ldots$ & Male. & Hernando & Mar. 30, 1879 & J. W. Milner. & $\begin{array}{r}m m \\
490\end{array}$ & $\begin{array}{l}m m \\
200\end{array}$ & $\begin{array}{c}m m \\
159\end{array}$ & $\begin{array}{l}m m \\
30\end{array}$ & $\begin{array}{l}m m \\
209\end{array}$ & $\begin{array}{l}m m \\
120\end{array}$ \\
\hline U.S.N.M. $90137^{1}$.. & ...do. & Punta Ras- & Mar. 10,1883 & C. W. Ward . & 498 & 194 & 167 & 32 & 217 & 122 \\
\hline U.S.N.M. $152839^{1}$. & ...do & Florida... &,- 1895 & W.F.Webb. & 488 & 186 & 152 & 32 & 211 & 117 \\
\hline U.S.N.M. 175531 & .... do & $\begin{array}{l}\text { Fort Gard- } \\
\text { ner. Fla. }\end{array}$ & Mar. 9, 1901 & E. A. Mearns & 500 & 185 & 153 & 30.5 & 213 & 116 \\
\hline U.S.N.M. $82329^{1}$.. & ...do. & $\begin{array}{l}\text { Near Oyster } \\
\text { Bay, Fla } 2\end{array}$ & Mar. -, 1881 & C. W. Ward. & 518 & 198 & 166 & 32 & 225 & 129 \\
\hline$\underset{99101.1}{\operatorname{Am} . \text { Mus. N. H. }}$ & ...do. & $\begin{array}{l}\text { Near Micco, } \\
\text { Fla. }\end{array}$ & Apr. 8,1898 & $\begin{array}{l}\text { L. A. Fuer- } \\
\text { tes. }\end{array}$ & 492 & 181 & 152.5 & 31 & 198 & 115 \\
\hline$\underset{49599.1}{\text { Am. Mus. N. H. }}$ & $\ldots$ do & $\begin{array}{l}\text { Suwa nee } \\
\text { River, La- } \\
\text { faye t } t \text { e }\end{array}$ & Mar. 21, 1890 & $\begin{array}{l}\text { F. M. Chap- } \\
\text { man. }\end{array}$ & 504 & 188.5 & 159.5 & 32 & 211 & 119 \\
\hline J. Dwight $24596^{1}$. & ...do. & Seven Oaks, & Apr. 6,1901 & & 493 & 195 & 140.5 & 30.5 & 195 & 118 \\
\hline U.S.N.M. $175423^{1}$. & & $\begin{array}{l}\text { Lake Hatch- } \\
\text { ne-haw, } \\
\text { Fla. }\end{array}$ & Feb. 14, 1901 & E.A.Mearns. & 503 & 197 & 167 & 31 & 232 & 121 \\
\hline $\begin{array}{l}\text { Am. Mus. N. H. } \\
39085 .\end{array}$ & $\begin{array}{l}\text { Male(?), } \\
\text { juv. }\end{array}$ & $\begin{array}{r}\text { E a s t e r n } \\
\text { peninsula, } \\
\text { opposite } \\
\text { Micco,Fla. }\end{array}$ & Mar. 6,1889 & $\begin{array}{l}\text { F. M. Chap- } \\
\text { man. }\end{array}$ & 460 & 163.5 & 150 & 31 & 202 & 109 \\
\hline J. Dwight $24597^{1}$. & Male. & $\begin{array}{l}\text { MeIntos h } \\
\text { Co., Ga. }\end{array}$ & Mar. 27,1890 & $\begin{array}{c}\text { W. W. Wor- } \\
\text { thington. }\end{array}$ & 486 & 182 & 161 & 30 & 200 & 119 \\
\hline
\end{tabular}


Measurements of specimens of Ardea herodias wardi-Continued.

Museum and No. Sex. Locality.

A. E. and O. Bangs Male.... St. Marys, 3068.1

Am. Mus. N. H. $79706 .^{1}$

Am. Mus. N. H. 79705.1

U.S.N.M. $184944^{1}$

U.S.N.M. $71162 \ldots$.... do....

C y p res s S w a mp, near m'til of White

$\mathrm{R}$ iver, s o u th west err Knox Co., Ind.

U.S.N.M. 71161

U.S.N.M. 84576 .

U.S.N.M. 71168 .

do.....

. 71165 . do

$\Delta \mathrm{m}$. Mus. N. H. ... do ........ do .....

J. Dwight 12358 .

Henry Co., 4 miles north of Hillsboro, Iowa.

U.S.N.M.72836 ... [Male]... Kansas..

U.S.N.M.1755321.. Female . Fort Gardner, Fla.

D rigg's $\mathrm{K}$ iss i mmee River, Fla,

Fort Bassinger, Fla.

Northern Brevard Co., Fla..

Near Micco,

Apr. 8,1898

Am. Mus. N. H. 99102.1

Am. Mus. N. H. 36981.1

Am. Mus. N. H. Female, $\mathrm{T}$ a $\mathrm{r}$ p o $\mathrm{n}$ 50613

J. Dwight 11902... Female . $\begin{gathered}\text { Ossabaw Is- } \\ \text { land, Ga. }\end{gathered}$ juv. Springs, Fla. U.S.N.M.39031.... ... do.... Hiltonhead, S. C.

U.S.N.M. 71169....... do... C y p res s S w a mp, near m'th of White $\mathrm{R}$ ive $\mathrm{r}$, south western Knox Co, Ind.

U.S.N.M. 72837.... [Female] ILount Carmel, IIl.

U.S.N.M. 126114 San Mateo, Fla.

U.S.N.M. 89966

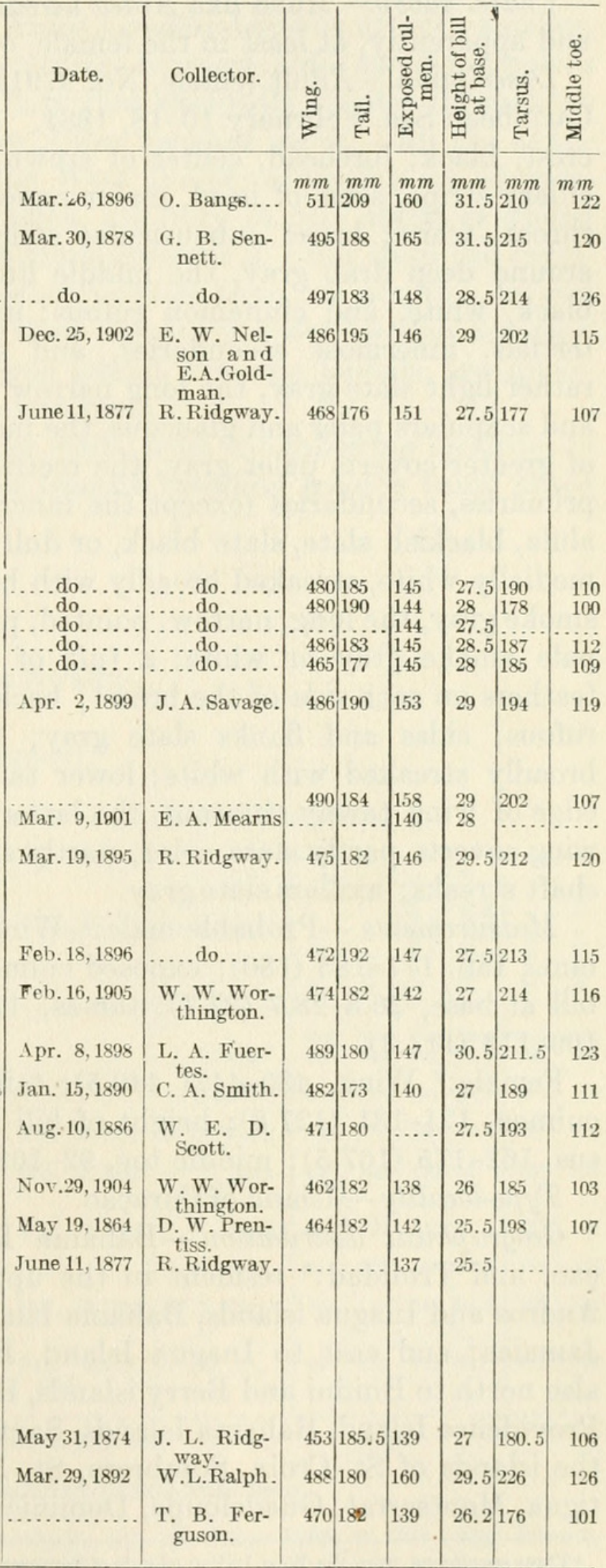


ARDEA HERODIAS ADOXA, new subspecies.

Chars. subsp.-Much like Ardea herodias herodias, but paler above, and apparently, at least in the female, of somewhat smaller size.

Description.-Adult female, No. 97914, U.S.N.M.; Curaçao Island, Caribbean Sea, February 10-18, 1884. Sides of crown, and occipital crest, black; forehead, center of crown, short vertical crest, superciliary stripe, sides of head, malar region, chin, and center of upper throat, white; broad subauricular stripe pale drab gray; neck all around deep drab gray, the middle line of foreneck streaked with black, white, and cinnamon rufous; upper surface, including tail, tertials, innermost secondaries, and superior secondary 'coverts, rather light slate gray, the long narrow plumaceous feathers of back and scapulars paler and glaucous, the median coverts and outer webs of greater coverts paler gray, the rectrices brownish on inner webs; primaries, secondaries (except the innermost), primary coverts, and alula, blackish slate, slate black, or dull black; jugulum smoke gray, medially white, streaked broadly with blackish slate, slate gray, and smoke gray, the long, narrow, pointed, plume-like feathers terminally pale smoke gray or white; a tuft of black, partly white-striped feathers on each side of the breast; back of this a patch of cinnamon rufous; sides and flanks slate gray; breast and abdomen. black, broadly streaked with white; lower tail-coverts white; thighs and edge of wing tawny chestnut, the latter shading to chestnut; under wing coverts partly slate color, partly slate gray, some with whitish shaft streaks; axillars slate gray.

Measurements.-Probable male: ${ }^{1}$ Wing, 460-468 (average, 464.7) mm.; tail, 177-185 (180); exposed culmen, 134-150 (143); height of bill at base, 26.5-28.7 (27.7); tarsus, 178-187 (181.7); middle toe, 109-113 (111.3).

Female: ${ }^{2}$ Wing, 430-447( 440.5); tail, 168-172 (170.5); exposed culmen, 121-131 (127.8); height of bill at base, 24-26.5 (24.8); tarsus, 162-175 (167.5); middle toe, 92-108 (102).

Type-locality.-Island of Curaçao.

Geographical distribution-Bahama Islands, West Indies, Curaçao, and Trinidad: ${ }^{3}$ resident in the upper Tropical Zone, north to Andros and Inagua islands, Bahama Islands; west to Cuba; south to Jamaica; and east to Inagua Island, Bahama Islands. In winter also north to Bimini and Berry islands, Bahama Islands; east to New Providence Island, Bahama Islands, Santo Domingo, Porto Rico, and the islands of St. Croix, Sombrero, St. Bartholemew, Barbuda, Antigua, Montserrat, Guadeloupe, Dominica, Martinique, Barbados, St.

1 Three specimens, from the West Indian islands of Sombrero, Guadeloupe, and Grenada.

2 Four specimens, from Inagua Island (Bahama Islands), Guadeloupe Island, and Jamaica, West Indies

${ }^{3}$ No specimens examined, but probably this form. 
Vincent, Carriacou, and Grenada; south to the Isle of Pines, Curaçao, and Trinidad.

This race is of the size of Ardea herodias herodias or even smaller, and in color is somewhat intermediate between Ardea herodias herodias and Ardea herodias wardi. It is thus decidedly smaller throughout and rather darker above than Ardea herodias wardi. All the available immature birds from the West Indies, excepting an adventitiously stained one from Jamaica, are fully as pale above as young Ardea $h$. wardi, and are even more different in color from the corresponding stage of Ardea herodias herodias than are adults. In size these immature birds are less than immature Ardea herodias herodias. Two breeding birds from Inagua Island, Bahama Islands, are apparently just like other West Indian birds.

Specimens examined number eight, from the following localities in the West Indies and Bahama Islands, breeding records being noted by an asterisk:

Sombrero Island.

Curaçao Island.

Jamaica.-Spanishtown.

Grenada Istand.-Telescope Swamp.

Guadeloupe Istand.

Inagua Island (Bahama Islands).*

Measurements of specimens of Ardea herodias adoxa.

\begin{tabular}{|c|c|c|c|c|c|c|c|c|c|c|}
\hline Museum and No. & Sex. & Locality. & Date. & Collector. & $\stackrel{80}{\leftrightarrows}$ & Еีं & 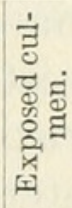 & 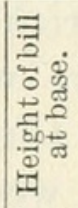 & 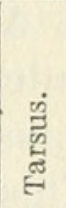 & 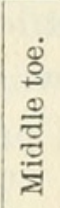 \\
\hline A m.M us. N.H. & & $\begin{array}{l}\text { Somb re ro } \\
\text { Id., W.I. }\end{array}$ & & A. Julien. & $\begin{array}{l}m m \\
466\end{array}$ & $\begin{array}{l}m m \\
185\end{array}$ & $\begin{array}{l}m m \\
145\end{array}$ & $\begin{array}{c}m m \\
28.7\end{array}$ & $\begin{array}{l}m m \\
180\end{array}$ & $\begin{array}{l}m m \\
109\end{array}$ \\
\hline U.S.N.M. $114910^{1}$. & [ $\mathrm{Male}$ ] & Guadeloupe & & L. Guesde... & 468 & 178 & 134 & 26.5 & 178 & 112 \\
\hline U.S.N.M. $81141^{1}{ }^{1}$. & ...do...... & Grenada Id., & Nov. 29,1880 & J. G. Wells.. & 460 & 177 & 150 & 28 & 187 & 113 \\
\hline $\begin{array}{l}\text { Field Mus. N . H. } \\
33764^{1} \text {. }\end{array}$ & Female. & $\begin{array}{l}\text { Inagua Id., } \\
\text { Bah a m a }\end{array}$ & Mar. 28,1888 & $\begin{array}{l}\text { C. J. May- } \\
\text { nard. }\end{array}$ & 447 & 168 & 130 & 24 & 163 & 104 \\
\hline$\underset{337631}{\text { Field Mus. N. H. }}$ & ...do. & $\begin{array}{l}\text { 1siands. } \\
\text {...... do....... }\end{array}$ & Mar. 2,1891 & C. S. Winch. & 430 & 170 & 121 & 24 & 162 & 92 \\
\hline U.S.N.M. $108058{ }^{1}$. & [Female] & Guadeloupe & & L. Guesde... & 445 & 172 & 129 & 24.5 & 175 & 104 \\
\hline U.S.N.M. $30346^{1}$.. & $\begin{array}{l}\text { Female } \\
\text { juv. }\end{array}$ & S pan ish- & Dec. 10,1863 & W.T.March. & 440 & 172 & 131 & 26.5 & 170 & 108 \\
\hline U.S.N.M. 97914.. & & $\begin{array}{l}\text { Curaçao Is- } \\
\text { land.2 }\end{array}$ & $\begin{array}{l}\text { Feb. }{ }_{1884}{ }^{10-18,} \\
\end{array}$ & & 447 & 179 & 145 & 30 & 172 & 104 \\
\hline
\end{tabular}

1 Used in measurement averages on p. 544.

2 Type.

\section{ARDEA HERODIAS TREGANZAI Court.}

Ardea herodias treganzai Court, Auk, vol. 25, July, 1908, p. 291 (Egg Island, Great Salt Lake, Utah).

Chars. subsp.-Resembling Ardea herodias herodias, but upper parts and neck paler; also in size averaging very slightly larger.

$48702^{\circ}$-Proc.N.M.vol.43-12-35 
Measurements.-Total length (in flesh), 1100-1170 mm.; extent of wing, $1755-1845$.

Male: ${ }^{1}$ Wing, 445-493 (average, 471.7) mm.; tail, 162-192 (179.9); exposed culmen, 132-157 (144.3); height of bill at base, 26-31 (28); tarsus, 165-193 (181.6); middle toe, 99-112.5 (105.9).

Female: ${ }^{2}$ Wing, 440-475 (455.5); tail, 164-182 (174.2); exposed culmen, 120.5-150 (137.2); height of bill at base, 25-28 (26.1); tarsus, 157-183 (170.5); middle toe, 89-107.5 (100.7).

Type locality.--Egg Island, Great Salt Lake, Utah.

Geographical distribution.-Western United States to southwestern Mexico: In summer chiefly the Lower Austral, Upper Austral, and Transition zones, north to Douglas County, southern Wyoming; Grace, southern Idaho; probably also to Montana and eastern Washington; west to central Washington (probably); central Oregon (probably); Nevada; Colorado River near Riverside Mountain, and Pelican Island, Salton Sea, southeastern California; and the Pacific Ocean at the United States and Mexican boundary line; ${ }^{3}$ south to Gardner's Laguna, Salton River, northern Lower California; Guaymas, central western Sonora ${ }^{4}$ Santa Cruz River west of the Patagonia Mountains, Arizona; Fort Fillmore and Carlsbad, southern New Mexico; Tornillo Creek, near Boquillas, central western Texas; and probably also northern Chihuahua; east to Kerr County, central Texas (probably); Denver, central Colorado; Douglas County, eastern Wyoming; and probably eastern Montana. Winters from Texas and Arizona south to Manzanillo, Colima, Mexico. In migration wanders west to the Sacramento Valley, California, and east to Corpus Christi, Texas.

This western race is apparently just like Ardea herodias wardi in color, but is decidedly smaller throughout. It differs from Ardea herodias adoxa, of the West Indies, in somewhat paler upper parts and slightly larger size. It is, in fact, closer in characters to this than to either Ardea herodias herodias or Ardea herodias wardi. The juvenal plumage of Ardea herodias treganzai is distinguishable from that of Ardea herodias herodias by its decidedly paler upper parts and usually paler neck.

There seems to be no difference of consequence between birds from various parts of this bird's range, as above defined. An immature specimen from Brownsville, Texas (No. 30259, J. Dwight), is apparently this form; as is also a bird from Corpus Christi (No. 79707, Amer. Mus. Nat. Hist.), taken at the late date of April 11 (1889). We have only the head and part of the neck of an immature,

\footnotetext{
1 Fourteen specimens, from Arizona, New Mexico, Texas, Idaho, and California, including seven not examined by the writer, but measured by Mr. Joseph Grinnell.

2 Twenty-two specimens, from Arizona, Texas, Utah, Wyoming, Montana, Lower California, and Chihuahua, including seven not examined by the writer, but measured by Mr. Joseph Grinnell.

${ }^{3}$ Probably not breeding here.

4 Probably Ardea herodias treganzai; eggs in the United States National Museum.
} 
apparently non-breeding example from the Pacific Ocean at the Mexican and United States boundary line (No. 133774, U.S.N.M.), taken July 16, 1894, but it seems to belong to this race, notwithstanding that the form from San Diego, California, is not the same. No breeding examples from the San Joaquin Valley, or from the southern Sacramento Valley, California, have been available, and we therefore can not determine the summer resident of this region. A single specimen without date, from the Sacramento Valley (No. 9472, U.S.N.M.), is apparently Ardea herodias treganzai, but it is doubtless a migrant or a straggler.

Altogether, 36 specimens have been seen, from the localities given below, breeding records being followed by an asterisk:

Arizona.-Sonoyta River at Quitobaquita (Feb. 4); Fort Verde; Gila River, 7 miles below San Carlos; Santa Cruz River, west of Patagonia Mountains;* Fort Lowell; Tucson; San Bernardino Ranch; Colorado River at Monument 204 (Mexican boundary line).*

California.-Sacramento Valley; Pacific Ocean at the Mexican boundary line.

\section{Idaho.-Grace.*}

Montana.-Gallatin Station.

New Mexico.-Luna; San Luis Springs.*

Texas.-Fort Clark (Jan. 24); Corpus Christi; Brownsville (Feb. $22)$.

Wyoming.-Douglas.*

Chihuahua.-Rio Grande River near El Paso, Texas (Feb. 27); San Diego.

Colima.-Manzanillo.

Lower California.-Gardner's Laguna, Salton River.*

Sonora.-Cajon Bonito Creek, near the United States boundary line.

Measurements of specimens of Ardea herodias treganzai.

\begin{tabular}{|c|c|c|c|c|c|c|c|c|c|c|}
\hline Museum and No. & Sex. & Locality. & Date. & Collector. & $\stackrel{\infty}{\vdots}$ & Еีं & 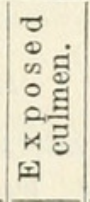 & 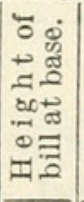 & 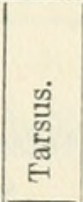 & 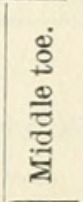 \\
\hline $\begin{array}{l}\text { U.S.N.M. } 204448^{1} . \\
\text { Am. Mus. N. H. }\end{array}$ & $\begin{array}{l}\text { Male,juv. } \\
\text { Male.... }\end{array}$ & $\begin{array}{l}\text { Luna, N. M. } \\
\text { Fort Verde, }\end{array}$ & $\begin{array}{r}\text { Sept. } 6,1908 \\
\ldots \ldots \ldots \ldots \ldots\end{array}$ & $\begin{array}{l}\text { C. Birdseye. } \\
\text { E. A. Mearns }\end{array}$ & \begin{tabular}{r|}
$m m$ \\
473 \\
477
\end{tabular} & $\begin{array}{l}m m \\
182 \\
188\end{array}$ & $\begin{array}{l}m m \\
138 \\
146.5\end{array}$ & $\begin{array}{l}m m \\
28.5 \\
28\end{array}$ & $\begin{array}{l}m m \\
179 \\
179\end{array}$ & $\begin{array}{l}m m \\
110 \\
100.5\end{array}$ \\
\hline $\begin{array}{l}\text { 51038.1 } \\
\text { A1034.1 } \\
\text { Mus. N. H. }\end{array}$ & ... & $\begin{array}{l}\text { Ariz. } \\
\ldots . \text { do........ }\end{array}$ & Dec. 26,1885 & .. do & 456 & 175 & 135 & 27 & 187 & 107.5 \\
\hline$\underset{51033.1}{\operatorname{Am} . \text { Mus. N. H. }}$ & ...do & ....do... & Sept. 13,1885 & ...do. & 471 & 184 & 143 & 29 & 188 & 105 \\
\hline Am. Mus. N. H. & ...do.. & $\begin{array}{l}\text { Corpus Chris- } \\
\text { ti, Tex. }\end{array}$ & Apr. 11,1889 & G. B. Sen- & 468 & 178 & 150 & 31 & 186 & 112.5 \\
\hline U.S.N.M. $9472^{1} \ldots$. & ... do & $\begin{array}{l}\text { Sacramento } \\
\text { V a l l e y, } \\
\text { Cal. }\end{array}$ & & $\begin{array}{l}\text { R. S. Will- } \\
\text { iamson. }\end{array}$ & 464 & 180 & 140 & 28 & 181 & 107 \\
\hline U.S.N.M. $204429^{1}$.. & ...do. & Grace,Idaho. & June 30,1907 & $\begin{array}{l}\text { C. D. Wal- } \\
\text { cott, jr. }\end{array}$ & 471 & 188 & 132 & 26 & 182 & 99 \\
\hline U.S.N.M. $131506^{1}$.. & $\begin{array}{c}\text { Female, } \\
\text { juv. }\end{array}$ & $\begin{array}{l}\text { Fort Lowell, } \\
\text { Ariz. }\end{array}$ & Nov. 7,1893 & E. A. Mearns & 460 & 176 & 140 & 26 & 178 & 98 \\
\hline
\end{tabular}

\footnotetext{
1 Used in measurement averages on p. 546.
} 
Measurements of specimens of Ardea herodias treganzai-Continued.

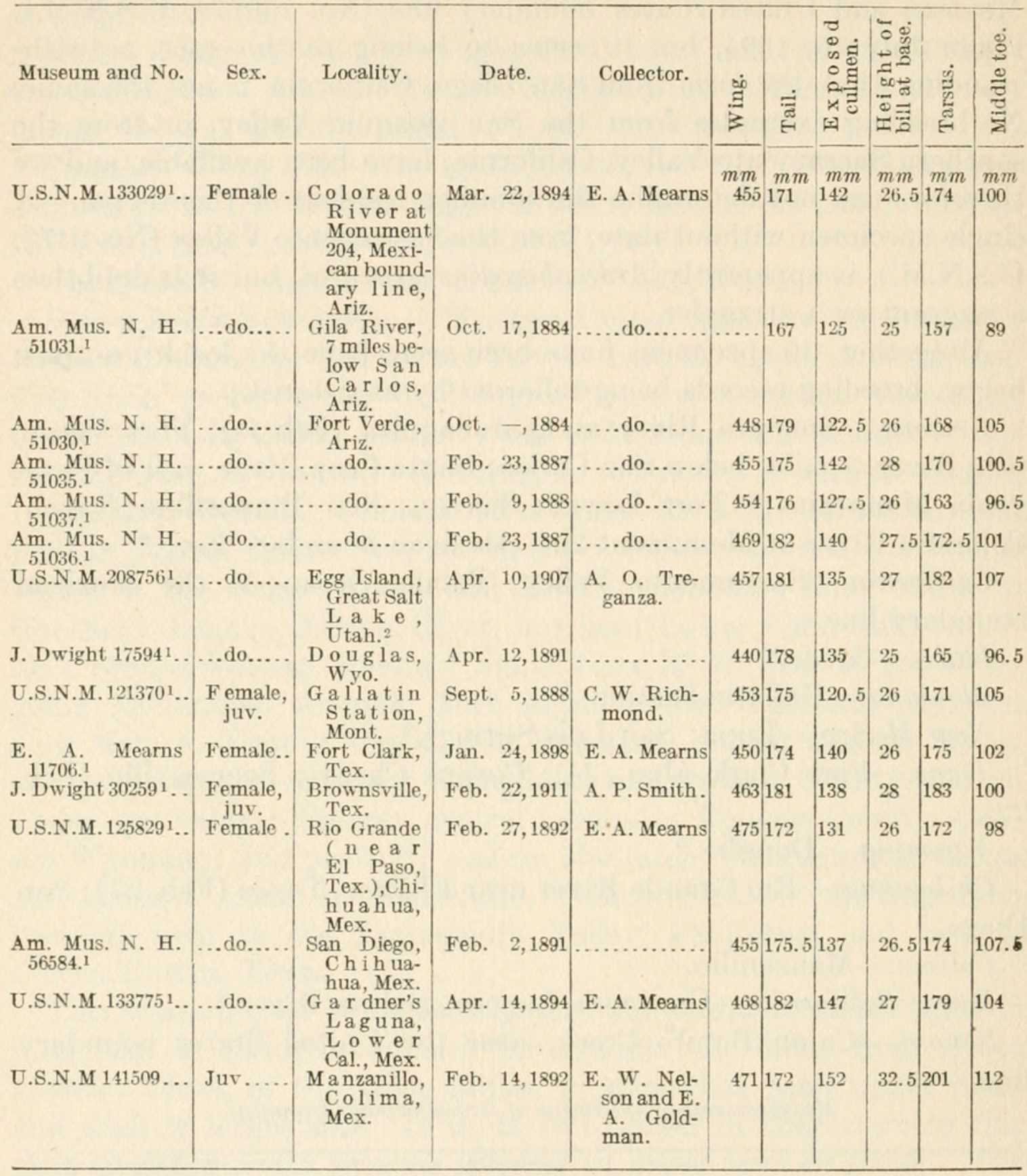

ARDEA HERODIAS SANCTILUCAE Thayer and Bangs.

Ardea herodias sancti-lucæ Thayer and Bangs, Proc. New Engl. Zool. Club, vol. 4, February 23, 1912, p. 83 (Espiritu Santo Island, Lower California, Mexico).

Chars. subsp.--Like Ardea herodias treganzai, but larger throughout, and with neck of a paler color.

Measurements.-Male: ${ }^{3}$ Wing, 475-500 (average, 485.5) mm.; tail, 179-195 (188.0); exposed culmen, 147-157 (150.8); height of bill at base, 28-30.5 (29.2); tarsus, 177-197 (189.6); middle toe, 106-115 (112.0).

Female: ${ }^{4}$ Wing, 455; tail, 175; exposed culmen, 132; height of bill at base, 26.5; tarsus, 169.5; middle toe, 99 .

1 Used in measurement averages on p. 546.

2 Type.
${ }^{3}$ Six specimens, from Lower California, Mexico.

1 One specimen, from Lower California. 
Type-locality.-Espiritu Santo Island, Lower California, Mexico.

Geographical distribution.--Upper Tropical Zone of southern Lower California, Mexico; south to San Jose del Cabo; north to San Jose Island. Probably resident throughout the year.

The present recently described form is easily separable from all the North American races, and seems most closely to resemble Ardea herodias cognata Bangs, from the Galapagos Islands. It may be distinguished from Ardea herodias adoxa, of the West Indies, by its much greater size throughout, and paler neck and upper parts; from Ardea herodias wardi by smaller size and more lightly colored neck. A bird taken in February is just as pale on neck and mantle as breeding specimens obtained in June. This race has a very limited distribution, the most limited, with possibly one exception, of any form of the group.

Seven specimens have been available, from the following localities, an asterisk indicating a breeding record:

Lower California.-Espiritu Santo Island;* San Jose del Cabo.

Measurements of specimens of Ardea herodias sanctilucae.

\begin{tabular}{|c|c|c|c|c|c|c|c|c|c|c|}
\hline Museum and No. & Sex. & Locality. & Date. & Collector. & $\stackrel{0}{\square}$ & हีं & 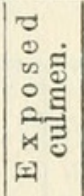 & 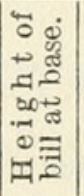 & 离 & 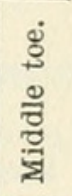 \\
\hline U.S.N.M. $33134^{1}$ & Male. & $\begin{array}{l}\text { San Jose del } \\
\text { Cabo,Low- } \\
\text { er Califor- } \\
\text { nia, Mex. }\end{array}$ & Feb.,- 1860 & $\begin{array}{l}\text { J. X antus } \\
\text { de Vesey. }\end{array}$ & $\begin{array}{c}m m \\
489\end{array}$ & $\begin{array}{c}m m \\
195\end{array}$ & $\begin{array}{c}m m \\
147\end{array}$ & $\begin{array}{l}m m \\
28\end{array}$ & $\underset{177}{m m}$ & $\begin{array}{c}m m \\
106\end{array}$ \\
\hline J.E.Thayer $18303^{1}$ & ... do. & $\begin{array}{c}\text { E spiritu } \\
\text { Santo Is- } \\
\text { land Low- } \\
\text { er Califor- } \\
\text { nia, Mex. }\end{array}$ & June 13,1910 & $\begin{array}{l}\text { W.W.Brown, } \\
\text { jr. }\end{array}$ & 500 & 195 & 147 & 28.5 & 193 & 114 \\
\hline $\begin{array}{l}\text { J.E.Thayer } 18305^{1} \\
\text { J.E.Thayer } 18304^{1}\end{array}$ & $\begin{array}{l}\text {..do. } \\
\ldots \text { do.. }\end{array}$ & $\begin{array}{l}\ldots . \text { do } \ldots . . . \\
\ldots . . \text { do } \\
\ldots\end{array}$ & $\begin{array}{l}\text { June } 14,1910 \\
\text { June } 15,1910\end{array}$ & $\begin{array}{l}\text {...do. } \\
\ldots \text { do. }\end{array}$ & $\begin{array}{l}498 \\
475\end{array}$ & $\begin{array}{l}190 \\
186\end{array}$ & $\begin{array}{l}151 \\
154\end{array}$ & $\begin{array}{l}29 \\
30\end{array}$ & $\begin{array}{l}192 \\
187.5\end{array}$ & $\begin{array}{l}112 \\
111\end{array}$ \\
\hline J.E.Thayer 183021 & ... do. & . do....... & June 16,1910 & do. & 475 & 183. & 148.5 & 29 & 197 & 114 \\
\hline J.E.Thayer $18301^{1}$ & $\ldots$ do. & ..... do...... & June 18,1910 & & 476 & 179 & 157 & 30.5 & 191 & 115 \\
\hline J.E.Thayer $18306^{1}$ & Female & ...... do...... & June 17,1910 & do & 455 & & 132 & 26.5 & 169.5 & 99 \\
\hline
\end{tabular}

\section{ARDEA HERODIAS COGNATA Bangs.}

Ardea herodias cognata Bangs, Proc. New Engl. Zool. Club, vol. 3, February 6, 1903, p. 100 (Indefatigable Island, Galapagos Islands).

Chars. subsp.--Similar to Ardea herodias sanctilucae, but wing and tarsus shorter, and bill thicker.

Measurements.-Probable male: ${ }^{3}$ Wing, $477 \mathrm{~mm}$.; tail, 192; exposed culmen, 159; height of bill at base, 32.5 ; tarsus, 174 ; middle toe, 114.

Probable female: ${ }^{4}$ Wing, 430-450 (440); tail, 171-172 (171.5); exposed culmen, 132-145 (138.5); height of bill at base, 29-30 (29.5); tarsus, 152-160 (156); middle toe, 97-101 (99).

1 Used in measurement averages on p. 548.

2 Type.
3 One specimen, from the Galapagos Islands.

1 Two specimens, from the same islands. 
Type-locality.-Indefatigable Island, Galapagos Islands.

Geographical distribution.-Galapagos Islands: Indefatigable, Duncan, Albemarle, and probably other islands of the archipelago. Doubtless permanently resident.

Notwithstanding its far isolated range, this subspecies much more closely resembles the pale races of North America and the West Indies than it does the very dark form of Mexico and Central America. It differs from Ardea herodias treganzai in paler neck, stouter bill, and shorter tarsus; from Ardea herodias wardi in lighter neck, stouter bill, and shorter wing and tarsus.

One of the specimens examined (No. 116138, U.S.N.M., Duncan Island) is not fully adult and is more brownish, somewhat darker above than the two adults. This race is doubtless peculiar to the Galapagos Archipelago, and probably occurs on many of the islands.

Only three examples have been seen, from the following localities, breeding records being distinguished by an asterisk:

Galapagos Islands.-Indefatigable Island; Duncan Island;* Albemarle Island.*

Measurements of specimens of Ardea herodias cognata.

\begin{tabular}{|c|c|c|c|c|c|c|c|c|c|c|}
\hline Museum and No. & Sex. & Locality. & Date. & Collector. & $\dot{80}$ & :ีं & 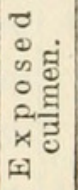 & 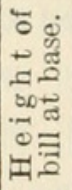 & 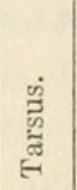 & 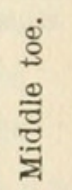 \\
\hline $\begin{array}{ll}\text { U.S.N.M. } & 189021^{1} \\
& \end{array}$ & [Male] .. & $\begin{array}{l}\text { Al b e m arle } \\
\text { Is l a n d, } \\
\text { Galapagos } \\
\text { Islands. }{ }^{2}\end{array}$ & Apr. 4,1902 & R. H. Beck. & $\begin{array}{r}m m \\
477\end{array}$ & $\begin{array}{c}m m \\
192\end{array}$ & $\begin{array}{c}m m \\
159\end{array}$ & $\begin{array}{l}m m \\
32.5\end{array}$ & $\begin{array}{c}m m \\
174\end{array}$ & $\underset{114}{m m}$ \\
\hline $\begin{array}{l}\text { U.S.N.M. 1161381 } \\
\text { A.E.and O. Bangs, } \\
\text { 12451. } 1\end{array}$ & $\begin{array}{l}{[\text { Female }]} \\
\ldots \text { do.... }\end{array}$ & $\begin{array}{l}\text { Duncan Is- } \\
\text { land, Gal- } \\
\text { apagos Is- } \\
\text { lands. } \\
\text { Ind efatiga- } \\
\text { b le Is- } \\
\text { land, Gala- } \\
\text { pagos Is- } \\
\text { lands.2 }\end{array}$ & $\begin{array}{l}\text { Apr. } 13,1888 \\
\text { Feb. } 16,1901\end{array}$ & R. H. Beck. & 430 & 172 & 132 & 29 & 152 & 101 \\
\hline
\end{tabular}

1 Used in measurement averages on p. 549 .

2 Type.

ARDEA HERODIAS HYPEROINA, new subspecies.

Chars. subsp.-Like Ardea herodias herodias in color, but in size decidedly greater throughout.

Description.-Type, adult male, No. 98486, U.S.N.M.; Baird, California, March 3, 1884; Charles H. Townsend. Sides of crown and long, slender, pointed occipital crest, black; forehead, center of crown, short vertical crest, superciliary stripe, malar region, cheeks, chin, and middle of upper throat, white; neck all around very deep drab gray, somewhat lighter anteriorly, the middle line of foreneck streaked with black, white, and cinnamon rufous; upper sur- 
face, including tail, tertials, innermost secondaries, and superior secondary coverts, slate-gray, the long, narrow, plumaceous feathers of back and scapulars paler and glaucous, the median coverts and outer webs of greater coverts paler gray, the rectrices more slaty on terminal portions; primaries, secondaries (except the innermost), primary coverts, and alula, blackish slate, slate black or dull black; jugulum deep smoke gray, medially white, streaked broadly with black and dull brownish slate, narrowly with cinnamon rufous and pale cinnamon, the long, narrow, pointed, plume-like feathers terminally pale drab gray, smoke gray, or whitish; a tuft of black, partly white-striped feathers on each side of the breast; back of this a small patch of cinnamon rufous; sides and flanks slate gray; breast and abdomen black, broadly streaked with white; under tail-coverts white; thighs and edge of wing deep cinnamon rufous, the latter in part more deeply colored, verging to chestnut; under wing-coverts slate color; axillars slate gray.

Measurements.-Male: ${ }^{1}$ Wing, 488-511 (average, 497.0) mm.; tail, 179-195 (186.5); exposed culmen, 139-148 (144.6); height of bill at base, 27.6-32 (29.5); tarsus, 180-198 (188.3); middle toe, 109-119 (112.4).

Female: ${ }^{2}$ Wing, 460-492 (473.0); tail, 171-180.5 (175.5); exposed culmen, 135-140 (137.7); height of bill at base, 25-29 (26.5); tarsus, 165-180 (170.3); middle toe, 94-109.6 (100.5).

Type-locality.-Baird, northern California.

Geographical distribution.-Pacific coast region of the United States, mainly in the Upper Austral and Transition zones: north to western Oregon; south to San Diego, southwestern California; east to San Gabriel, western California, and Baird, central northern California. Apparently a permanent resident throughout most if not all of its range, but wanders in winter west to the Farallon Islands, California, and east to St. John, Glenn County, California.

This new race differs much more from all the subspecies of Ardea herodias that are geographically near than it does from the far-distant typical form of the species. It may readily be distinguished from Ardea herodias treganzai by larger size and darker neck and upper parts, from Ardea herodias sanctilucae by much darker neck and mantle and average longer wing. There does not seem to be any constant difference in color between Ardea herodias hyperonca and Ardea herodias herodias, but the substantial difference in size serves well enough to separate them.

Two specimens from San Diego, California, taken respectively December 16, 1892 (No. 37088, Acad. Nat. Sci. Phila.), and April 24, 1862 (No. 4494, Mus. Vert. Zool. ${ }^{3}$ ), the latter apparently in the breeding 
season, are, in both size and color, essentially like other examples of the present form from northern California, although the first-mentioned bird is slightly paler, as would be natural from an area so near the range of Ardea herodias treganzai.

An immature winter example from St. John, Glenn County, California (No. 197862, U.S.N.M.), taken January 2, 1906, belongs apparently to this form; and the breeding bird of the whole Sacramento Valley may be the same. Two specimens collected by the United States exploring expedition under Captain Wilkes (Nos. 12670 and 15352, U.S.N.M.) and labeled simply "Oregon " must be referred to the present form, although rather darker above than birds from northern California. They are somewhat vergent toward Ardea herodias fannini, and probably came from near the mouth of the Columbia River-at least no farther north-since all the great blue herons we have seen from Washington are Ardea herodias fannini.

Specimens to the number of eight have been seen, from the localities given below:

California.-Humboldt Bay; St. John; San Diego; Baird; San Gabriel.

Oregon.-[No further locality specified.]

Measurements of specimens of Ardea herodias hyperonca.

\begin{tabular}{|c|c|c|c|c|c|c|c|c|c|c|}
\hline Museum and No. & Sex. & Locality. & Date. & Collector. & $\stackrel{00}{\Xi}$ & : & 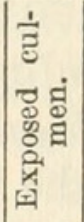 & 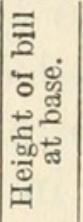 & 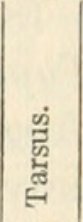 & 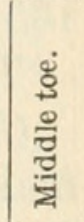 \\
\hline A.N.S.Phila.370881. & Male & San Diego, & Dec. 16,1892 & C. H. Marsh. & $\begin{array}{c}m a m \\
488\end{array}$ & $\begin{array}{l}m m \\
192\end{array}$ & $\underset{146}{m m}$ & $\begin{array}{c}m m \\
28.5\end{array}$ & $\begin{array}{c}m m \\
185\end{array}$ & $\begin{array}{c}m m \\
109\end{array}$ \\
\hline 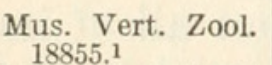 & ...do. & $\begin{array}{c}\text { Monterey } \\
\text { Bay, Cal. }\end{array}$ & Sept. 12,1910 & R. H. Beck.. & 497 & 180 & 147 & 30.5 & 193 & 113.2 \\
\hline $\begin{array}{l}\text { Mus. Vert. Zool. } \\
17244.1\end{array}$ & ...do.. & $\begin{array}{c}\text { S u i s u n } \\
\text { M a r shes, } \\
\text { S o lan o } \\
\text { Co., Cal. }{ }^{2}\end{array}$ & Dec. 28,1910 & $\begin{array}{l}\text { E. H. Cuth- } \\
\text { bert. } \\
\text { L. Kellogg. }\end{array}$ & 511 & 195 & 139 & 28.5 & 180 & 111.3 \\
\hline 1724 & & & Det. 0,1910 & 1. NenOEs. & & & & & & \\
\hline $107401^{1}$. & {$[$ Male $] ..$.} & $\begin{array}{c}\text { Hum boldt } \\
\text { Bay, Cal. }\end{array}$ & Dec. 7,1885 & $\begin{array}{l}\text { C. H. Town- } \\
\text { send. }\end{array}$ & 490 & 179 & 148 & 32 & 198 & 119 \\
\hline U S N M 084861 & $\ldots$ do...... & Baird, Cal. ${ }^{3}$. & Mar. 3,1884 & $\ldots . .$. do ...... & 500 & 193 & 141.5 & 30 & 192 & 110 \\
\hline & Male,juv.4 & $\begin{array}{l}\text { St. J o h n, } \\
\text { Glenn Co., } \\
\text { Cal. }\end{array}$ & Jan. 21,1906 & J. H. Gaut.. & 467 & 179 & 156 & 28.5 & 195 & 115 \\
\hline$\underset{4494.1}{\text { Mus. Vert. Zool. }}$ & Female. . & $\underset{\text { Cal.2 }}{\text { San Diego, }}$ & Apr. 24,1862 & J. G. Cooper. & 492 & 175 & 138 & 25.4 & 180 & 109.6 \\
\hline Am. Mus. N. H. & Female, & $\underset{\text { Cal.2 }}{\text { San Gabriel, }}$ & Sept. 13, 1888 & $\begin{array}{l}\text { E. C. Thur- } \\
\text { ber. }\end{array}$ & 460 & 180.5 & 135 & 25 & 165 & 98 \\
\hline $\begin{array}{l}\text { U.S.N.M. } 12670^{1} \ldots \\
\text { U.S.N.M } 15352 \ldots\end{array}$ & [Fen & Oregon... & & $\dddot{\mathrm{T}} \mathrm{R}$ & $\begin{array}{l}467 \\
479\end{array}$ & $\begin{array}{l}171 \\
182\end{array}$ & $\begin{array}{l}140 \\
146\end{array}$ & $\begin{array}{l}29 \\
28\end{array}$ & $\begin{array}{l}166 \\
177\end{array}$ & $\begin{array}{r}94 \\
104\end{array}$ \\
\hline U.S & & Baird, Cal... & Nov. 1,1883 & $\begin{array}{l}\text { C. H. Town- } \\
\text { send. }\end{array}$ & ...... & 204 & 135 & 26.5 & 171 & 103 \\
\hline
\end{tabular}

1 Used in measurement averages on p. 551 .

2 Not seen; measured by Mr. Joseph Grinnell.

3 Type.

4 Probably a female. 
ARDEA HERODIAS OLIGISTA, new subspecies.

Chars. subsp.-In color like Ardea herodias hyperonca, but length of wing very much less, bill more slender, and middle toe shorter.

Description.-Type, almost adult male, No. 135573, U.S.N.M.; San Clemente Island, California, August 26, 1894; Dr. Edgar A. Mearns. Sides of crown, and occipital crest, black; center of crown, vertical crest, malar region, chin, and middle of upper throat, white; sides of head drab-gray; neck all around deep smoke gray, the middle line of foreneck streaked with black, white, and cinnamon rufous; upper surface, including tail, tertials, innermost secondaries, and superior secondary coverts, slate gray, rather darker on middle of back, the median coverts and outer webs of most of greater coverts paler gray, the rectrices terminally inclining to slate color or blackish slate; primaries, secondaries (except the innermost), primary coverts, and alula, blackish slate, slate black or dull black; jugulum smoke gray, medially white, streaked broadly with deep smoke gray and slate color, anteriorly washed with cinnamon rufous, the long, narrow, pointed, plume-like feathers terminally pale gray or whitish; a tuft of black, partly white-striped feathers on each side of the breast; back of this a patch of cinnamon rufous; sides and flanks slate gray; breast and abdomen striped, black, slate gray, and white; lower tail-coverts white; thighs and edge of wing cinnamon rufous, the latter darker along secondaries, somewhat mixed with white at base of primaries; under wing-coverts slate color, with some edgings of chestnut; axillars slate gray.

Measurements.-Male: ${ }^{1}$ Total length (in flesh), $1160 \mathrm{~mm}$; wing, 433; tail, 187; exposed culmen, 149; height of bill at base, 26; tarsus, 184; middle toe, 101.

Type-locality.- San Clemente Island, Santa Barbara Islands, California.

Geographical distribution.--Upper Austral Zone on the Santa Barbara Islands, California: San Clemente Island; also Santa Cruz, San Nicholas, Anacapa, Santa Catalina, and probably other islands of the group. Apparently a permanent resident.

Although the type is the only specimen, it is so very much smaller than any example of the mainland form, Ardea herodias hyperonca, that it seemingly can not belong to the same race. It has a shorter wing than even Ardea herodias herodias. From Ardea herodias treganzai and Ardea herodias sanctilucae it differs in much shorter wing and darker neck and mantle.

The type-specimen, though evidently full grown, is not quite adult in color of plumage, which it shows in its brown forehead and fore part of crown; in duller gray and in slight ochraceous admixture 
on neck; some grayish in the tufts on each side of the breast, and on feathers of breast and abdomen; and the not fully developed long, narrow, plumaceous feathers of back and scapulars.

This race is probably confined to the Santa Barbara Islands, as the species is said to be resident there. Mr. G. Willett writes ${ }^{1}$ that the great blue heron occurs and doubtless breeds in isolated pairs on most, if not all of these islands, but that he has actually seen nests on only Santa Catalina and Anacapa islands. Although, of course, we know certainly of the subspecific status of only the bird on San Clemente Island, yet the published records of the great blue heron from Santa Catalina, Santa Cruz, and San Nicholas islands belong without much doubt under this form.

ARDEA HERODIAS FANNINI Chapman.

Ardea herodias fannini Chapman, Bull. Amer. Mus. Nat. Hist., vol. 14, April 15, 1901, p. 87 (Skidegate, Graham Island, Queen Charlotte Islands, British Columbia).

Chars. subsp.-Similar to Ardea herodias hyperonca, but wing, exposed culmen, and middle toe shorter, the tarsus still more so; tail longer; upper parts and neck darker.

Measurements.-Male: ${ }^{2}$ Wing, 472-492 (average, 480.7) mm.; tail, 192-195 (193.7); exposed culmen, 124.5-137 (132.8); height of bill at base, 27-28 (27.3); tarsus, 153-167.5 (160.2); middle toe, 97-107 (100.7).

Female: ${ }^{3}$ Wing, 456-486 (466.3); tail, 186-194 (189); exposed culmen, 123-129.5 (126.8); height of bill at base, 24.5-26 (25.5); tarsus, 146-158 (153); middle toe, 88.5-96 (93.2).

Type-locality.-Skidegate, Graham Island, Queen Charlotte Islands, British Columbia.

Geographical distribution.-Pacific coast region of northern North America, mostly in the Transition and Canadian zones: north to Hope, Cook Inlet, Alaska; south to Cape Flattery, northwestern Washington; and the Nisqually Flats, central western Washington. Permanently resident, except perhaps in the northernmost part of its range.

This dark northwestern race is readily separable from Ardea herodias herodias by its longer wing and tail, decidedly shorter tarsus, average shorter culmen, much darker neck and upper parts. It is so very different from Ardea herodias treganzai, by reason of its longer wing and tail, shorter tarsus, middle toe, and culmen, and very much darker neck and upper parts, that close comparison is unnecessary. The very short tarsus of this subspecies, actually as well as relatively, distinguishes it from all the other forms of the species.

\footnotetext{
1 In letter.

2 Three specimens, from Washington and British Columbia.

$s$ Three specimens, from Alaska and British Columbia.
} 
An immature specimen from Admiralty Island, Alaska (No.187748, U.S.N.M.), is very dark on neck and back, but this difference is apparently not more than individual. This race is confined, so far as known, to a narrow strip of country along the coast and to the islands, from the State of Washington to Alaska.

Ten specimens have been examined, from the subjoined localities, breeding records being followed by an asterisk:

Alaska.-Admiralty Island.

British Columbia.- Sumas (Feb. 1); Sooke Lake; Skidegate, Graham Island (Queen Charlotte Islands); Victoria.

Washington.-Cape Flattery; Nisqually Flats.*

Measurements of specimens of Ardea herodias fannini.

\begin{tabular}{|c|c|c|c|c|c|c|c|c|c|c|}
\hline Museum and No. & Sex. & Locality. & Date. & Collector. & $\stackrel{00}{\Xi}$ & न्ञ & 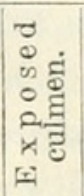 & 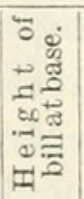 & 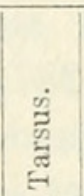 & 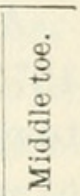 \\
\hline A.N.S.Phila. $30616^{1}$ & Male. & Nisqu a $11 \mathrm{y}$ & Apr. 19,1892 & S. N. Rhoads. & $\begin{array}{c}m m \\
492\end{array}$ & $\begin{array}{c}m m \\
194\end{array}$ & $m m$ & $\operatorname{mm}_{28}$ & $\underset{167.5}{m m}$ & $\begin{array}{l}m m \\
97\end{array}$ \\
\hline$\underset{73572.1}{\operatorname{Am} . \text { Mus. N. H., }}$ & ...do.... & Sooke Lake, & Mar. 26,1900 & . & 478 & 192 & 137 & 27 & 153 & 98 \\
\hline A. E. and $O$. & ...do... & Sumas, B.C & Mar. 22,1903 & A. C. Brooks. & 472 & 195 & 124.5 & 27 & 160 & 107 \\
\hline U.S.N.M.187748 $1 .$. & $\begin{array}{c}\text { Female, } \\
\text { juv. }\end{array}$ & $\begin{array}{c}\text { Near Killis- } \\
\text { noo, Ad- } \\
\text { miralty Is- } \\
\text { land, Alas- }\end{array}$ & Sept. 25, 1903 & $\begin{array}{l}\text { W. H. Os- } \\
\text { good. }\end{array}$ & 486 & 194 & 128 & 24.5 & 155 & 96 \\
\hline $\begin{array}{l}\text { A. E. and O. } \\
\text { Bangs, 11159. }\end{array}$ & Female. & Sumas, B. C. & Feb. 1,1895 & A. C. Brooks. & 457 & 187 & 129.5 & 26 & 158 & 95 \\
\hline$\underset{74294.1}{\operatorname{Am} . \text { Mus. N. H. }}$ & [Female] & $\begin{array}{c}\text { Skidegate, } \\
\text { Q u e e n } \\
\text { Charlot t e } \\
\text { Islands, B. } \\
\text { C. } 2\end{array}$ & Feb. -, 1901 & $\begin{array}{l}\text { J. R. Swan- } \\
\text { ton. }\end{array}$ & 456 & 186 & 123 & 26 & 146 & 88.5 \\
\hline U.S.N.M. 4524. & & $\begin{array}{c}\text { Cape Flat- } \\
\text { terv, Wash. }\end{array}$ & & $\begin{array}{l}\text { W. P. Trow- } \\
\text { bridge. }\end{array}$ & 474 & 190 & 122 & 26 & 149 & 91 \\
\hline J. Dwight & & Victoria, B.C. & & & 472 & 189 & 126 & 27.5 & 160 & 91 \\
\hline
\end{tabular}

ARDEA HERODIAS LESSONII Wagler.

Ardea lessonii Wagler, Isis, 1831, p. 531 (Mexico).

Chars. subsp.-Resembling Ardea herodias fannini, but wing and tail shorter; exposed culmen, middle toe, and especially tarsus, longer; upper parts and neck a little lighter in color.

Measurements.-Male: ${ }^{3}$ Wing, 470-475 (average, 471.8) mm.; tail, 174-187 (181.3); exposed culmen, 138-154 (146); height of bill at base, 30-31 (30.3); tarsus, 185-191 (188.5); middle toe, 101-113 (108.3).

Female: ${ }^{4}$ Wing, 441-464 (455.7); tail, 173-182 (177); exposed culmen, 111-138 (125.7); height of bill at base, 23-27.5 (25.7); tarsus, 157-178 (166.3); middle toe, 93-111 (100).

1 Used in measurement averages on p. 554.

2 Type.

3 Four specimens, from the States of Jaliseo and Michoacan, Mexico, and from Panama.

"Three specimens, two from the Mexican States of Mexico and Chihuahua, and one from "Mexico." 
Type-locality.-Valley of Mexico, State of Mexico, Mexico. ${ }^{1}$

Geographical distribution.-Mexico, Central America, and northern South America, breeding in the Upper Austral, Lower Austral, Upper Tropical, and Lower Tropical zones: north to southern Sinaloa; Colonia Garcia, northwestern Chihuahua; and Tamaulipas; southeast through all of Mexico to Guatemala, Nicaragua, Costa Rica, Panama, and Merida, Venezuela. Resident all the year practically throughout its range, except perhaps in South America.

From Ardea herodias hyperonca the present subspecies may be distinguished by its shorter wing, darker neck and upper parts; from Ardea herodias herodias by longer wing and tail and darker neck and upper surface. The young in first plumage are like those of Ardea herodias fannini, but are somewhat lighter on neck and upper parts.

A single adult from Chihuahua City, Chihuahua, Mexico (No. 187287, U.S.N.M.), taken February 22, 1904, inclines very little toward Ardea herodias treganzai; and a juvenal from Tamaulipas, exact locality unknown (No. 30477, Acad. Nat. Sci. Phila.), appears also to be $A$. $h$. lessonii. Specimens examined from Nicaragua and Costa Rica are all in juvenal plumage, and without adults it is difficult to place them with certainty. They are no darker than juvenal Ardea herodias herodias, and might suggest the existence of another race in this region, were it not that a juvenal of typical Ardea herodias lessonii, from Patzcuaro, Michoacan, Mexico, is precisely the same in color. It may be, therefore, that the young of Ardea herodias lessonii is not darker than the same age of Ardea herodias herodias, although the adult is decidedly so. At any rate, until more satisfactory material is available, the bird of Central America down to Costa Rica, inclusive, must pass as Ardea herodias lessonii.

A single bird in juvenal plumage from Empire, in the Canal Zone, Panama, is practically identical with young birds from Mexico, Nicaragua, and Costa Rica, except for being rather darker, more slaty, on the neck. Another bird, from Panama is, however, darker above than any adult specimen of Ardea herodias lessonii that we have seen; and with more material, the Panama bird may be separable subspecifically from that of Mexico. Since the above-mentioned example is in rather an interesting phase of plumage, almost adult, and very much more brownish than either young or adult normally are, the following description may be of interest:

Almost adult male, No. 230144, U.S.N.M., Biological Survey collection; Fort Lorenzo, Panama, June 21, 1911; E. A. Goldman. Forehead and sides of sinciput clove brown; sides of crown, and occipital crest, black; superciliary stripe pale gray; center of crown, vertical crest, postocular region, malar region, chin and middle of upper throat, white; subauricular region and sides of throat light 
brownish gray; neck all around deep brownish gray, somewhat paler anteriorly, the median line of foreneck streaked with black, clove brown, cinnamon rufous, and white; upper surface, including tail, tertials, innermost secondaries, and superior secondary coverts, slaty brown, the middle of back darker, the median coverts and outer webs of greater coverts lighter; primaries, secondaries (except the innermost), primary coverts, and alula, brownish slate black; jugulum deep brownish gray, medially white, broadly streaked with blackish slate, slate gray, and smoke gray, narrowly with chestnut and cinnamon, the long, narrow, pointed, plume-like feathers terminally white; a tuft of brownish black, mostly white-striped, chestnut-streaked feathers on each side of the breast; back of this a small patch of deep cinnamon rufous; sides and flanks brownish slate; breast and abdomen white, broadly streaked with slate black and slate gray, narrowly with chestnut and cinnamon rufous; lower tail-coverts white; thighs and edge of wing cinnamon rufous, the latter partly mixed with white; lining of wing, including axillars, slate color, but some of the coverts with chestnut edgings.

It thus differs from the fully adult bird in its brownish forehead and sides of sinciput; deep brownish neck and upper parts; rusty edgings of upper wing-coverts; much mixture of white in the edge of the wing; slaty tufts on the sides of the breast; mostly gray breast and abdomen; and the imperfect development of the dorsal, scapular, and jugular plumaceous feathers.

From the date of capture of the previously mentioned Chihuahua example, and from other winter dates given in the list of specimens below, it is evident that the present race is resident throughout the year over most if not all of its range.

The subspecific name which is here applied has been entirely lost sight of for many years, and the writer is indebted to Mr. Ridgway for calling his attention to this long-forgotten description. This Ardea lessonii of Wagler ${ }^{1}$ is based on a great blue heron from "Mexico," and there is no reasonable doubt concerning its proper application to the present form.

Twelve examples of this race have been seen, from localities as follows, breeding records being indicated by an asterisk:

Chihuahua.-Colonia Garcia (Feb. 22).

Jalisco.-Ocotlan (Dec. 28); La Barca (Dec. 18).

Mexico (State).-San Mateo (Dec. 9).

Michoacan.-Patzcuaro.*

Tamaulipas.-[No locality specified.]

Costa Rica.-Lipurio.

Nicaragua.-San Juan del Sur (Jan. 5).

Panama.-Fort Lorenzo;* Empire. 
Measurements of specimens of Ardea herodias lessonii.

\begin{tabular}{|c|c|c|c|c|c|c|c|c|c|c|}
\hline Museum and No. & Sex. & Locality. ' & Date. & Collector. & $\dot{30}$ & न्ँં & 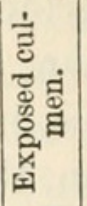 & 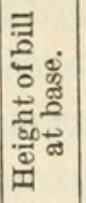 & 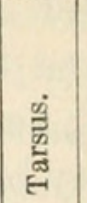 & 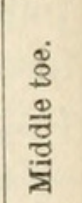 \\
\hline U.S.N.M. $187291^{1}$. & Male.... & $\begin{array}{l}\text { La Barca, } \\
\text { J a lisc o, } \\
\text { Mex. }\end{array}$ & Dec. 18,1903 & $\begin{array}{l}\text { E. W. Nel- } \\
\text { son and } \\
\text { E.A.Gold- } \\
\text { man. }\end{array}$ & $\begin{array}{r}m m \\
470\end{array}$ & $\begin{array}{c}m m \\
184\end{array}$ & $\underset{138}{m m}$ & $\begin{array}{l}m m \\
30\end{array}$ & $\begin{array}{c}m m \\
188\end{array}$ & $\begin{array}{c}m m \\
101\end{array}$ \\
\hline U.S.N.M. $184943^{1}$. & $\ldots$ do.... & $\begin{array}{l}\text { Ocot la n, } \\
\text { J a } 1 \text { isco, } \\
\text { Mex. }\end{array}$ & Dec. 28,1902 & ..... do...... & 472 & 187 & 143 & 30 & 190 & 109 \\
\hline U.S.N.M. $193839^{1}$. & $\begin{array}{c}\text { Ma } 1 \text { e, } \\
\text { juv. }\end{array}$ & $\begin{array}{l}\text { Patzcu a r o, } \\
\text { Michoacan, } \\
\text { Mex. }\end{array}$ & July 15,1904 & $\ldots$ do...... & 475 & 180 & 154 & 31 & 185 & 113 \\
\hline U.S.N.M. $66344^{2}$. . & ...do.... & $\begin{array}{l}\text { L ip u rio, } \\
\text { C o s t a } \\
\text { Rica. }\end{array}$ & & W. M. Gabb. & 435 & 165 & 128 & 25 & 166 & 101 \\
\hline U.S.N.M. $230144^{1}$. & $\begin{array}{l}\text { Male, vix } \\
\text { ad. }\end{array}$ & $\begin{array}{l}\text { Fort Loren- } \\
\text { zo, Pana- } \\
\text { ma. }\end{array}$ & June 21,1911 & $\begin{array}{l}\text { E. A. Gold- } \\
\text { man. }\end{array}$ & 470 & 174 & 149 & 30 & 191 & 110 \\
\hline U.S.N.M. $187287^{1}$ & [Female] & $\begin{array}{l}\text { Colonia Gar- } \\
\text { cia, Chi- } \\
\text { hua h ua, } \\
\text { Mex. } \\
\text { Mexico....... }\end{array}$ & Feb. 22, 1904 & J. H. Gaut. . & 462 & 182 & 128 & 27.5 & 178 & 111 \\
\hline M.C.Z. $56889^{1} \ldots \ldots$ & Female. & $\begin{array}{l}\text { San Mateo, } \\
\text { Mexico, } \\
\text { Mex. }\end{array}$ & Dec. 9,1910 & $\begin{array}{l}\text { Wr. W. Brown, } \\
\text { jr. }\end{array}$ & 464 & 176 & 138 & 26.5 & 164 & 96 \\
\hline A.N.S.Phila. 30477 & Juv..... & $\begin{array}{l}\text { Tamaulipas, } \\
\text { Mex. }\end{array}$ & & L. B. Couch. & 449 & 173 & 130.5 & 26.5 & 169 & 97.5 \\
\hline A. E. and O. Bangs & & Costa Rica.. & & $\begin{array}{l}\text { C. F. Under- } \\
\text { wood. }\end{array}$ & 462 & 171 & 139.5 & 26.5 & 181 & 103 \\
\hline U.S.N.M. $89783 \ldots$ & Juv ..... & $\begin{array}{l}\text { San Juan del } \\
\text { Sur, Nica- } \\
\text { ragua. }\end{array}$ & Jan. 5,1883 & $\begin{array}{l}\text { C. C. Nut- } \\
\text { ting. }\end{array}$ & 445 & 179 & 140 & 26.5 & 175 & 98 \\
\hline
\end{tabular}

1 Used in measurement averages on p. 555.

2 Evidently not full grown.

KEY TO THE SUBSPECIES OF ARDEA HERODIAS.

a. Colors very dark; general tone of mantle slate color or deep brownish slate.

b. Tarsus shorter (that of male less than $170 \mathrm{~mm}$.)...........Ardea herodias fannini.

$b^{1}$. Tarsus longer (that of male more than $170 \mathrm{~mm}$.)........ Ardea herodias lessonii. $a^{1}$. Colors not very dark; general tone of mantle slate gray or lighter.

$b$. Wing of male averaging more than $480 \mathrm{~mm}$.

c. Upper parts darker; mantle slate gray.............. Ardea herodias hyperonca. $c^{1}$. Upper parts paler; mantle lighter gray.

d. Smaller (tarsus of male less than $200 \mathrm{~mm}$.; wing of male averaging less than

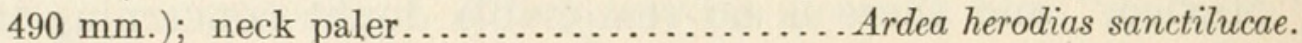

$d^{1}$. Larger (tarsus of male usually more than $200 \mathrm{~mm}$.; wing of male averaging more than $490 \mathrm{~mm}$.); neck darker............... Ardea herodias wardi.

$b^{1}$. Wing of male averaging less than $480 \mathrm{~mm}$.

c. Upper surface lighter.

d. Neck paler; bill heavier (height at base usually not less than $29 \mathrm{~mm}$.).

Ardea herodias cognata.

$d^{1}$. Neck darker; bill more slender (height at base usually less than $29 \mathrm{~mm}$.).

$c^{1}$. Upper surface darker.

Ardea herodias treganzai.

d. Smaller (wing of male less than $440 \mathrm{~mm}$.)...........Ardea herodias oligista. $d^{1}$. Larger (wing of male more than $440 \mathrm{~mm}$.).

$e$. Mantle lighter; size somewhat smaller (wing of female averaging less than

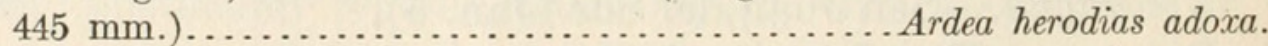

$e^{1}$. Mantle darker; size somewhat larger (wing of female averaging more than $445 \mathrm{~mm}$.$) .......................... Ardea herodias herodias.$ 


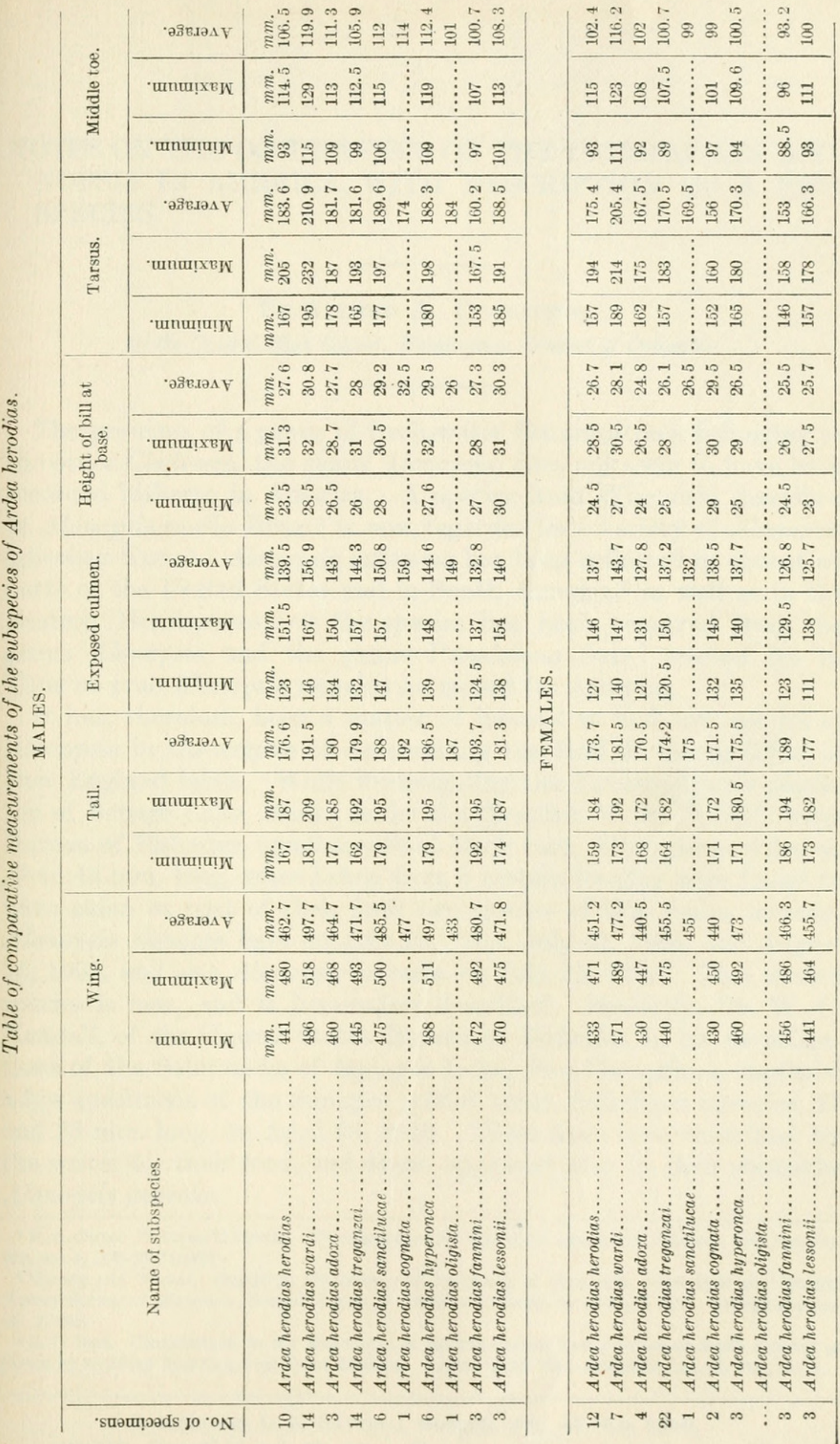




\section{$2 \mathrm{BHL}$ Biodiversity Heritage Library}

Oberholser, Harry C. 1912. "A revision of the forms of the great blue heron (Ardea herodias Linnaeus)." Proceedings of the United States National Museum 43(1939), 531-559. https://doi.org/10.5479/si.00963801.1939.531.

View This Item Online: https://www.biodiversitylibrary.org/item/32782

DOI: https://doi.org/10.5479/si.00963801.1939.531

Permalink: https://www.biodiversitylibrary.org/partpdf/12263

\section{Holding Institution}

Smithsonian Libraries

\section{Sponsored by}

Smithsonian

\section{Copyright \& Reuse}

Copyright Status: NOT_IN_COPYRIGHT

This document was created from content at the Biodiversity Heritage Library, the world's largest open access digital library for biodiversity literature and archives. Visit BHL at https://www.biodiversitylibrary.org. 